ARTICLE

\title{
Arabidopsis SABRE and CLASP interact to stabilize cell division plane orientation and planar polarity
}

Stefano Pietra ${ }^{1}$ Anna Gustavsson ${ }^{1}$, Christian Kiefer ${ }^{1}$, Lothar Kalmbach ${ }^{1} \uparrow$, Per Hörstedt ${ }^{2}$, Yoshihisa Ikeda ${ }^{1, \dagger}$, Anna N. Stepanova ${ }^{3}$, Jose M. Alonso ${ }^{3} \&$ Markus Grebe ${ }^{1}$

The orientation of cell division and the coordination of cell polarity within the plane of the tissue layer (planar polarity) contribute to shape diverse multicellular organisms. The root of Arabidopsis thaliana displays regularly oriented cell divisions, cell elongation and planar polarity providing a plant model system to study these processes. Here we report that the SABRE protein, which shares similarity with proteins of unknown function throughout eukaryotes, has important roles in orienting cell division and planar polarity. SABRE localizes at the plasma membrane, endomembranes, mitotic spindle and cell plate. SABRE stabilizes the orientation of CLASP-labelled preprophase band microtubules predicting the cell division plane, and of cortical microtubules driving cell elongation. During planar polarity establishment, sabre is epistatic to clasp at directing polar membrane domains of Rho-of-plant GTPases. Our findings mechanistically link SABRE to CLASP-dependent microtubule organization, shedding new light on the function of SABRE-related proteins in eukaryotes.

\footnotetext{
${ }^{1}$ Umeå Plant Science Centre, Department of Plant Physiology, Umeå University, SE-90 187 Umeå, Sweden. ${ }^{2}$ Umeå Core Facility Electron Microscopy, Umeå University, SE-90 187 Umeå, Sweden. ${ }^{3}$ Department of Genetics, North Carolina State University, Raleigh, North Carolina 27695, USA. †Present addresses: Department of Plant Molecular Biology, University of Lausanne, UNIL-Sorge, Biophore Building, 1015 Lausanne, Switzerland (L.K.); Centre of the Region Haná for Biotechnological and Agricultural Research, Department of Molecular Biology, Faculty of Science, Palacký University Olomouc, Šlechtitelů 11, 78371 Olomouc, Czech Republic (Y.I.). Correspondence and requests for materials should be addressed to M.G. (email: markus.grebe@umu.se).
} 
$\mathrm{D}$ iverse multicellular organisms coordinate the polarity of single cells within the plane of the tissue layer ${ }^{1-5}$. This type of tissue polarity is commonly referred to as planar polarity ${ }^{1,2,4}$. The establishment of planar polarity is intensively studied for animal epithelia where Drosophila wing-hair polarization provides an invaluable model system ${ }^{1,2}$. Similarly, plants like Arabidopsis display planar polarity of coordinated polar hair outgrowth from root epidermal cell $s^{3,4}$ and orientation of leaf epidermal hairs (trichomes) ${ }^{5}$. The upstream molecular mechanisms that direct planar polarity, however, differ substantially between animals and plants. In Drosophila, Wingless and its homologue dWnt4 act as long-range polarizing cues upstream of the Frizzled planar cell polarity pathway ${ }^{\text {, }}$ which transmits directional information to downstream effectors such as small Rho/Rac-type family GTPases $^{1,2}$. By contrast, plants lack genes with similarity to Wnts or to core components of the Frizzled planar cell polarity pathway. Instead, planar polarity of root epidermal cells is coordinated by a tissue concentration gradient of the plant hormone auxin ${ }^{4,7}$. This gradient instructs the coordinated polar placement of hairs and of Rho-of-plant (ROP) GTPases that mark the initiation of planar polarity of root hair positioning before morphological hair outgrowth ${ }^{4,7}$. ROP2/4 localization provides the earliest read-out for polar hair initiation 8,9 and regulation of ROP activity spatially restricts polar root hair initiation ${ }^{9,10}$. During morphogenesis of leaf pavement cells, auxin can activate ROP2/4 and ROP6 to differentially organize the actin and tubulin cytoskeleton, respectively ${ }^{11,12}$. Actin and tubulin are also required for planar polarity of root hair initiation ${ }^{13,14}$, but cytoskeletal dynamics and their regulation remain uncharacterized during this process. Similarly, the sole microtubule-associated protein known to be required for planar orientation of leaf trichomes is the CLIP170-associated protein (CLASP) from Arabidopsis ${ }^{15}$. Further, CLASP mediates orientation of cell division planes in $\operatorname{roots}^{16,17}$, modulates the abundance $e^{18,19}$ as well as the polarity of PIN2 protein in root cortical cells ${ }^{19}$, and CLASP homologues regulate cellular asymmetries in other eukaryotes ${ }^{20}$. At the molecular level, CLASP homologues associate with and stabilize the plus ends of dynamic microtubules ${ }^{20}$, stabilize microtubule arrays during cell division ${ }^{15,16,20}$ and promote microtubule growth around cell edges contributing to the generation of plant cortical microtubule arrays ${ }^{21}$.

Here we report new functions for the Arabidopsis SABRE $(S A B)$ gene, mechanistically linking its action to CLASP-mediated microtubule organization. We thus unravel new interactions contributing to the framework of cell division plane orientation and planar polarity formation in plants. Our findings may help to elucidate the function of uncharacterized SAB-like proteins found throughout eukaryotes and their potential function in cytoskeletal organization during cell division, cell morphogenesis and its coordination.

\section{Results}

kreuz und quer affects cell and planar polarity. The root hairs of Arabidopsis regularly emerge from the outer membrane of epidermal cells and coordinately initiate close to, albeit not completely at, the root tip-oriented (basal) ends of cells (Fig. 1a). As knowledge about genes contributing to planar polarity in plants remains limited, we performed a genetic screen for root hairpositioning mutants to identify additional factors. This led to the identification of the recessive kreuz und quer (kuq) mutant generated by fast neutron mutagenesis. kuq displayed a more random distribution of hair position towards both the basal and the apical ends of cells (Fig. 1b,c). We also observed the localization of polar ROP protein patches at the outer membrane of root hair cells before morphological hair outgrowth. Unlike wild type (Fig. 1d), kuq mutant cells often showed more basal or apical localization of ROP (Fig. 1e-g). In contrast, the polar distribution of PEN3-GFP (Supplementary Fig. S1a,b) that broadly defines the outer membrane ${ }^{22,23}$ as well as the apical polarity of the PIN2 auxin efflux carrier (Supplementary Fig. S1c,d) remained largely unaffected in root epidermal cells of kuq (Supplementary Fig. S1b,d). More strikingly, the polarity of GFP-BASL ${ }^{24}$, which almost invariably localizes to basal membranes and to nuclei when ectopically expressed in wild-type root epidermal cells (99.8\% basal, $0.2 \%$ of cells apical localization; $n=514$ ) was significantly more often switched to apical ends of epidermal cells in kuq $(8.6 \%$ of cells with apical localization; $n=187$; $P=5.1 \times 10^{-9}$ by Fisher's exact test; Supplementary Fig. S1e, f). These findings suggest that $k u q$ affects basal membrane polarity and basal bias of planar polarity rather than outer lateral membrane polarity in the root epidermis.

Leaf trichome orientation provides another model for the analysis of both cell and planar polarity. Arabidopsis wild-type trichomes predominantly carry three branches, and the first branch, initiating closest to the leaf surface, is oriented towards the proximal end of the leaf ${ }^{5,15}$ providing a planar polarity readout. Intriguingly, orientation of the first branch proved much more variable in the kuq mutant than in the wild type (Fig. $1 \mathrm{~h}-\mathrm{j}$ ), revealing defective planar polarity of trichome orientation. At the cellular level, $k u q$ mutant leaves showed two-branched trichomes more often than wild-type leaves (Fig. 1k,l; for quantitative analysis see below) and sometimes displayed irregular placement of branches along the trichome stalk (Fig. $1 \mathrm{~m}$ ). The reduction in branch number and the ectopic placement of branches on trichomes in kuq indicated a cell polarity defect in the initiation and placement of trichome branches. Interestingly, reduced trichome branch number has been reported for Arabidopsis mutants defective in tubulin and microtubule-associated proteins ${ }^{25}$. Hence, $k u q$ affects cell and planar polarity in leaf and root epidermis, causing phenotypes characteristic of microtubule cytoskeleton mutants.

kuq is allelic to SABRE. We mapped the kuq mutation to a 480 -kb interval on chromosome 1 by positional cloning (Fig. 2) and analysed phenotypes of T-DNA insertion lines in candidate genes within this interval. Homozygous seedlings from 9 out of 11 T-DNA insertion lines in one of these genes, SABRE (SAB) (At1g58250; Fig. 2) displayed phenotypes indistinguishable from that of kuq, whereas two showed similar but weaker seedling root and adult shoot defects (Figs 2 and 3a). Sequencing of the genomic $S A B$ coding region in $k u q$ identified a single-nucleotide deletion at position 6,592 (of 7,824), predicted to induce a nonsense mutation generating a premature stop codon (Fig. 2). Other $S A B$-mutant alleles have been described to display anisotropic expansion of root cortical cells ${ }^{26-28}$ or an enhanced response to phosphate starvation ${ }^{29}$. More detailed analysis of three $S A B$ T-DNA insertion lines, SALK 108709 (sab-5), SALK 52995 (sab-6) and SALK 123771 (sab-7) uncovered reduced epidermal cell length and defective planar polarity of root-hair position similar to that of the kuq mutant (Fig. 3b-e). Complementation analyses revealed that these three lines were allelic to $k u q$ and to each other and they are, therefore, in the following referred to as $s a b^{k u q}, s a b-5, s a b-6$ and $s a b-7$. The sab-7 mutant carrying an insertion into the second last exon of the $S A B$ gene (Fig. 2) was somewhat less affected, identifying it as a hypomorphic allele (Fig. 3a,b,e). Reverse transcription PCR (RT-PCR) analyses revealed that neither sab-5, sab-6 nor sab-8 (SALK 12897) produced a full-length transcript, in contrast to $s a b^{k u q}$ for which a transcript could be detected when using primers spanning the 

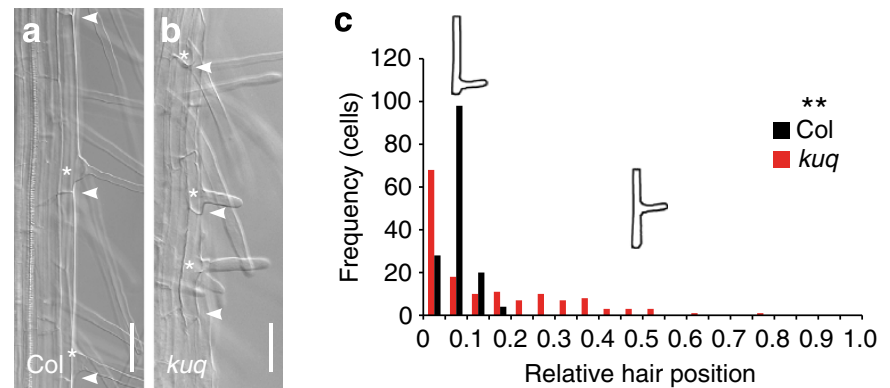

g
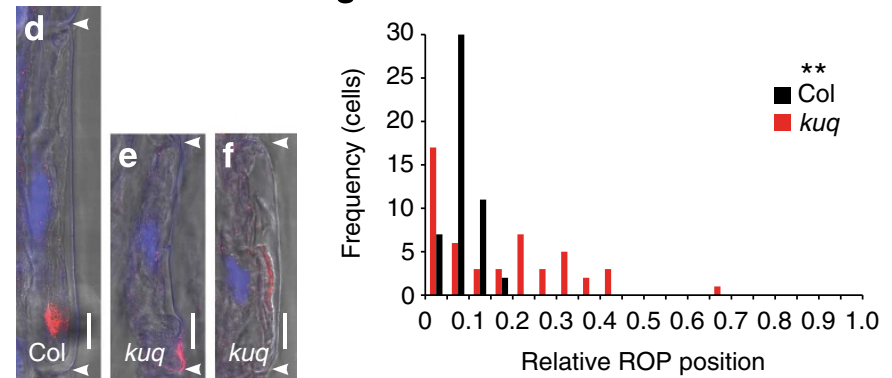

Relative ROP position
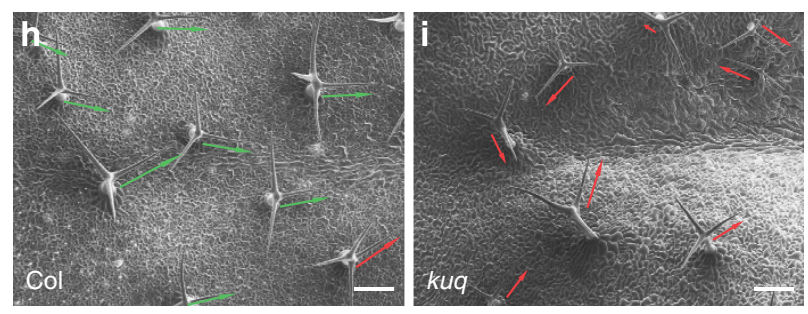

j

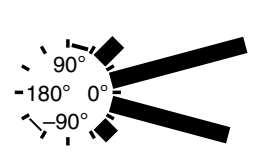

Col

$-90^{\circ}$

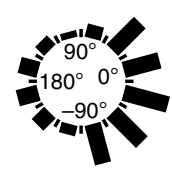

kuq
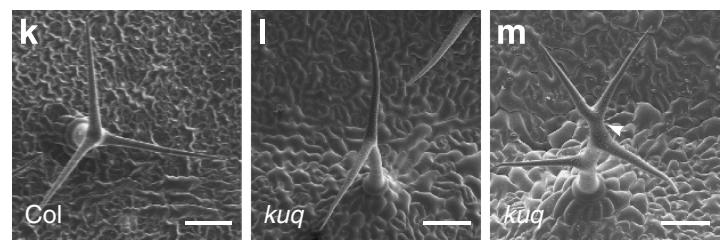

Figure 1 | kuq mutation affects epidermal cell and planar polarity. (a,b) Hair-forming root epidermal cells of 5-day-old (a) wild-type Columbia (Col) and (b) kuq seedlings. Arrowheads, apical and basal ends of cells in $\mathbf{a}, \mathbf{b}$ and $\mathbf{d}-\mathbf{f}$. Asterisks indicate sites of hair formation in a and $\mathbf{b}$. (c) Quantitative analysis of planar polarity phenotypes as relative root hair position in Col and kuq. Number of cells in classes (frequency) with hair positions are indicated between basal $(0)$ and apical (1) ends of cells ${ }^{\star *} P=0.000$ Col versus kuq, determined by non-parametric, two-sample Kolmogorov-Smirnov (K-S) test, $n=150$ cells from 30 roots per genotype). (d-f) anti-ROP (red) immunofluorescence in Col, (e,f) kuq epidermal cells, combined with DAPI-stained nuclei (blue) and brightfield microscopy image. ( $(\mathbf{g})$ Quantitative analysis of planar polarity phenotypes of ROP protein patch positions along apical-basal axis of epidermal cells in Col and kuq. Number of cells in classes (frequency) showing ROP positions between basal (0) and apical (1) ends is shown ( ${ }^{\star \star} P=0.000$ Col versus kuq by K-S test, $n=50$ cells per genotype, from $10 \mathrm{Col}$ and 19 kuq roots). (h,i,k-m) Scanning electron microscopy (SEM) images of leaf trichomes of (h,k) Col, (i,I, $\mathbf{m})$ kuq-mutant leaves. (h,i) Planar polarity of first trichome branch orientation along proximo-distal leaf axis (arrows). Distal ends of leaves are to the left, proximal ends to the right. Deviation of $<30^{\circ}$ and $>30^{\circ}$ from proximal orientation is indicated by green and red arrows, respectively. (j) Quantitative analysis of first trichome branch orientation from SEM images of Col and kuq. $0^{\circ}$ indicates proximal orientation, $180^{\circ}$ distal orientation of trichome branches. ( ${ }^{\star \star} P<0.001$ Col versus kuq by F-test, $n=174$ trichomes per genotype). ( $(\mathbf{k}-\mathbf{m})$ Trichome branching phenotypes of (k) Col and $(\mathbf{I}, \mathbf{m})$ kuq. Note, (I) trichomes with two branches or ( $\mathbf{m}$ ) additional ectopically placed branch in kuq (arrowhead). For statistical analysis, see Fig. 5 a. Scale bars, $50 \mu \mathrm{m}(\mathbf{a}, \mathbf{b}), 10 \mu \mathrm{m}(\mathbf{d}-\mathbf{f}), 200 \mu \mathrm{m}(\mathbf{h}, \mathbf{i}), 100 \mu \mathrm{m}(\mathbf{k}-\mathbf{m})$.

region including the single-nucleotide deletion (Fig. $3 \mathrm{f}-\mathrm{h}$ ). We subsequently employed $s a b-5$ in parallel to or instead of $s a b^{k u q}$ because $s a b-5$ carried the T-DNA insertion closest to the $5^{\prime}$-end of the $S A B$ coding region (Fig. 2), did not express full-length $S A B$ complementary DNA (cDNA) (Fig. $3 \mathrm{f}-\mathrm{h}$ ) and displayed phenotypic defects similar to sab ${ }^{k u q}$ (Fig. 3a-c, Supplementary Fig. S2a-1). Thus, cloning of $s a b^{k u q}$ revealed a central role for $S A B$ in cell and planar polarity.

SABRE-like proteins in other eukaryotes. Our sequencing of $S A B$ wild-type cDNA amplified from Arabidopsis roots experimentally verified the presence of an 8,347-bp transcript encoding a 2,607 amino acid protein identical to the predicted protein sequence Atlg58250.1 (ref. 29). Depending on the transmembrane helix prediction algorithm employed, $\mathrm{SAB}$ is predicted to contain one (TMHMM, Pred-TMR), two (TMpred) or three transmembrane domains (TopPred2, HMMTOP). All programs predict the first six to eight amino-terminal amino acids preceding the first transmembrane domain to be cytosolic. This indicates that SAB contains an internal, hydrophobic starttransfer sequence rather than an $\mathrm{N}$-terminal cleavable signal peptide and that $S A B$ may be a transmembrane protein rather than a secreted protein. Proteins with sequences related to the predicted 2,607 amino acid SAB protein are found throughout diverse eukaryotes (Fig. 4a). In Arabidopsis thaliana, KINKY
POLLEN (KIP) shares the highest sequence identity with $\mathrm{SABRE}^{28}$. Although plant species harbour either one or two $S A B$ like genes in their genomes, all animal genomes analysed, including the human and mouse genomes, possess a singlecopy gene encoding a protein of similar size with highest sequence identity restricted to two larger protein regions (Fig. 4b). To date, none of the genes related to $S A B$ from nonplant eukaryotes has been functionally characterized. However, mutants defective in the Arabidopsis KIP gene and the closely related ABERRANT POLLEN TRANSMISSION1 (APT1) gene from maize are defective in pollen tube growth ${ }^{28,30}$. APT1, like $S A B$, codes for a predicted 2,607 amino acid protein ${ }^{30}$. When transiently expressed in tobacco pollen, a carboxy-terminal 108 amino acid peptide from APT1 fused to green fluorescent protein (GFP) localizes to the Golgi apparatus ${ }^{30}$. The peptide has been annotated as a Golgi-body localization domain in the conserved domain database ${ }^{31}$. However, although $\mathrm{SAB}$ is a predicted membrane protein with the $\mathrm{N}$ terminus oriented towards the cytosol, the biological and molecular function of SAB and related proteins as well as the subcellular localization of full-length proteins remain to be addressed in any eukaryote.

$S A B-C L A S P$ interaction in planar polarity. As the SAB sequence did not provide information about the molecular function of the protein, we addressed the mechanism of SAB 


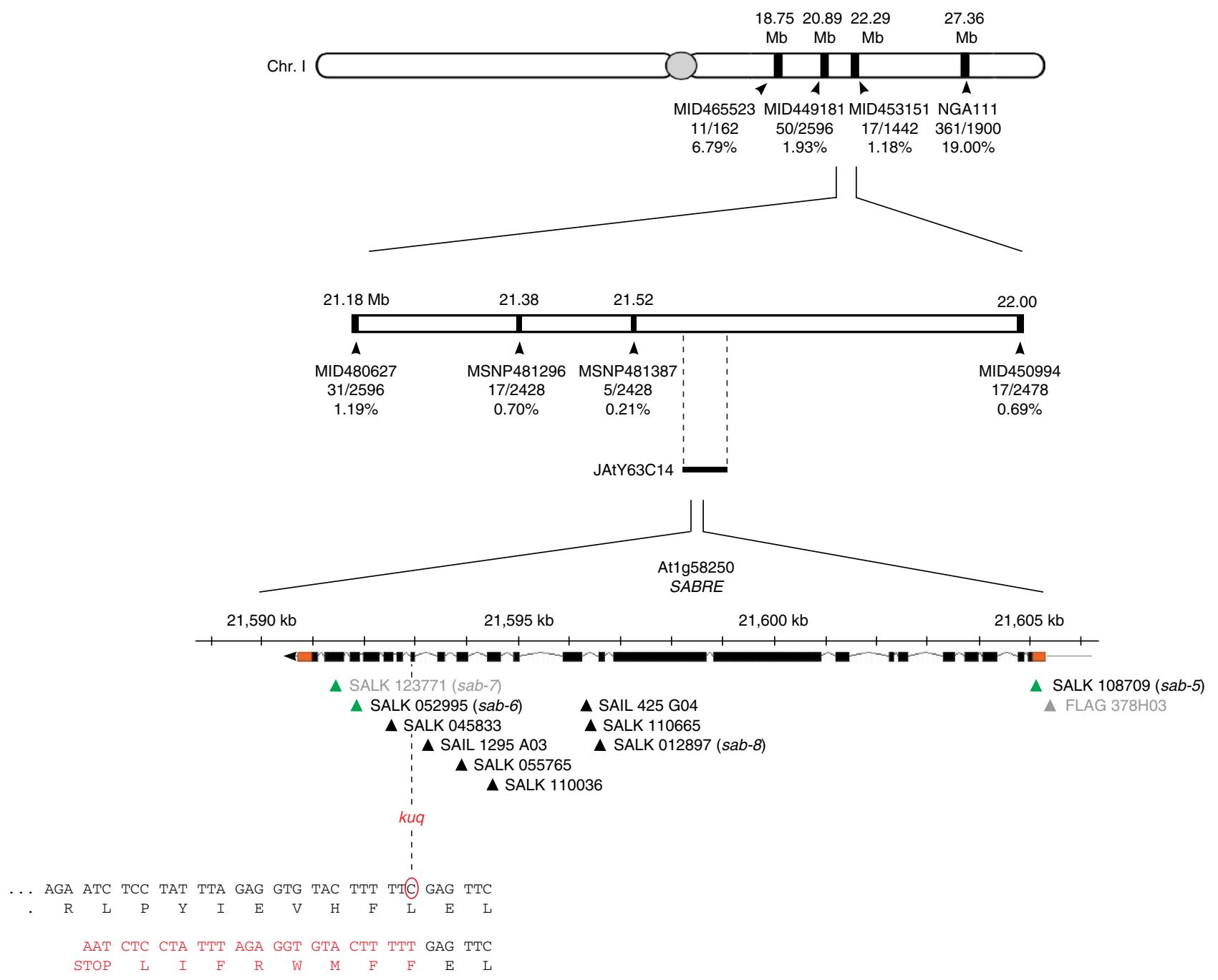

Figure 2 | kuq causes a single-nucleotide deletion in the SABRE gene. Map-based identification of the kuq mutation in the SAB gene. Simple-sequencelength polymorphism (SSLP; NGA111), custom-made SSLPs (Monsanto Insertion/Deletion polymorphism; MID) and custom-made cleaved amplified polymorphic sequence (CAPS; MSNP) markers are indicated with respective position, recombinant/total chromosomes and recombination frequency. Digits of MID and MSNP markers correspond to polymorphism accession number in Monsanto Arabidopsis Polymorphism Collection (http:// www.arabidopsis.org/browse/Cereon/index.jsp). Note the 480-kb mapping interval containing JAtY63C14 transformation-competent bacterial artificial chromosome carrying the $S A B$ gene. Insertion positions of 11 SAB T-DNA lines analysed are indicated by arrowheads below the gene structure. Black boxes, exons; lines, introns; orange boxes, untranslated regions. T-DNA lines in black, homozygous seedlings phenotypically indistinguishable from kuq mutants; T-DNA lines in grey, weaker phenotype. T-DNA lines with green arrowheads, tested for allelism to kuq by complementation test. Red circle, cytosine deletion in kuq inducing a premature stop codon in 17th exon of $S A B$; upper rows, wild-type coding sequence and amino-acid translation; lower rows, kuq.

action by analysing its genetic interactions. The combination of trichome branching and orientation defects observed in $s a b$ mutants had been described for clasp mutants ${ }^{15}$. We therefore generated a sab-5;clasp-1 double mutant and observed a strongly enhanced trichome branching defect, when compared with the respective single mutants or $s a b^{k u q}$ (Fig. 5a; Supplementary Fig. S3a,b), suggesting that $S A B$ and CLASP synergistically interact during placement of trichome branches. Similarly, root length and overall growth of sab-5;clasp-1 double-mutant seedlings was severely reduced compared with the single mutants, further pointing towards a synergistic interaction at this level (Fig. 5b; Supplementary Fig. S3c).

We next addressed whether CLASP acts on planar polarity of root hair placement and observed a clear basal shift of hair position in clasp-mutant alleles (Fig. 5c-e). Similarly, ROP protein patches were misplaced towards the basal-most ends of elongating clasp-mutant epidermal cells when compared with the wild type (Fig. $5 \mathrm{f}-\mathrm{h}$ ), demonstrating that CLASP acts on planar polarity before ROP positioning. Strikingly, ROP positioning in sab-5;clasp-1 double-mutant seedlings was indistinguishable from that of $s a b-5$ (Fig. $5 \mathrm{i}-\mathrm{k}$ ), suggesting that $s a b$ is epistatic to clasp during polar ROP positioning.

$S A B-C L A S P$ interaction during division plane orientation. The genetic interactions between $S A B$ and CLASP prompted us to investigate whether $S A B$ contributes to other processes that involve CLASP function, such as orientation of the cell division plane. Strikingly, $s a b-5-$ and $s a b^{k u q}$-mutant root meristems displayed strongly aberrantly orientated cell division planes when compared with the wild type (Fig. 6a,b; Supplementary Fig. S3d,e). Live imaging of microtubules and nuclear DNA co-visualized by mCherry-TUBULIN ALPHA-5 (mCherryTUA5) $)^{32,33}$ and HISTONE2B-YELLOW FLUORESCENT 


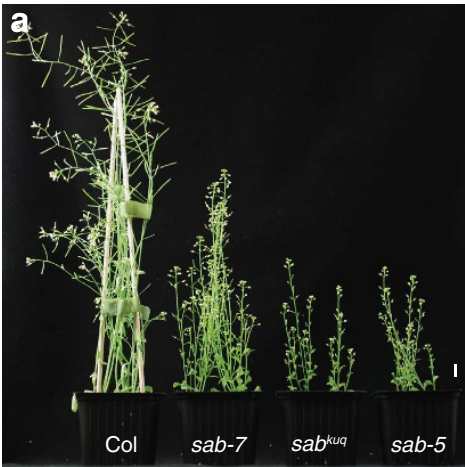

b

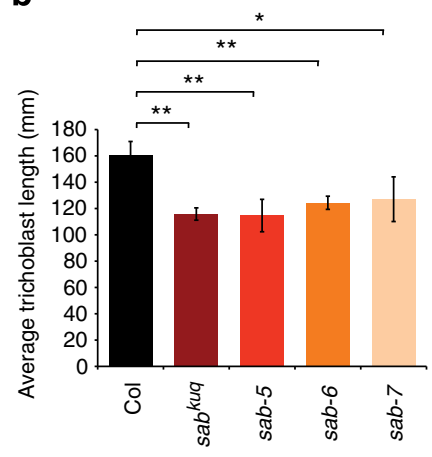

C

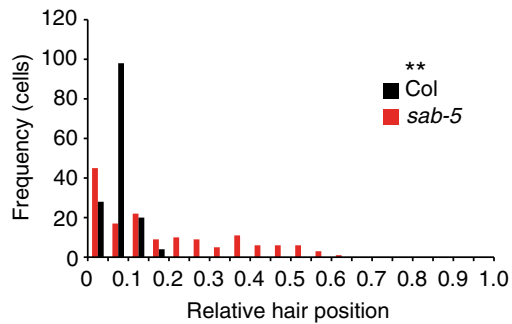

d

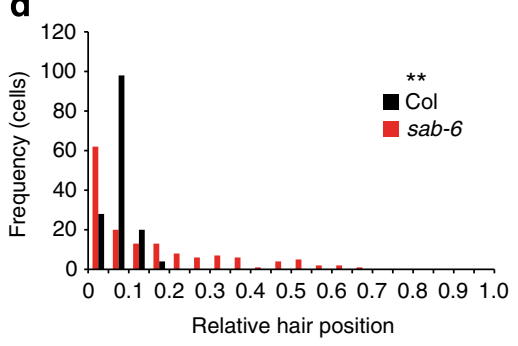

。

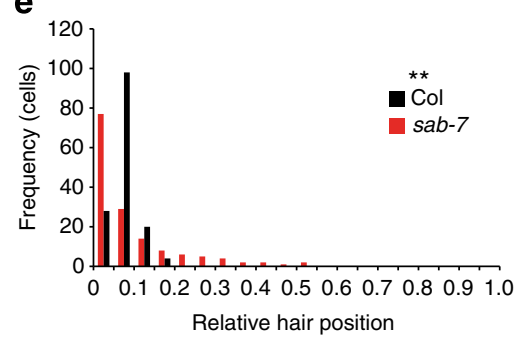

f

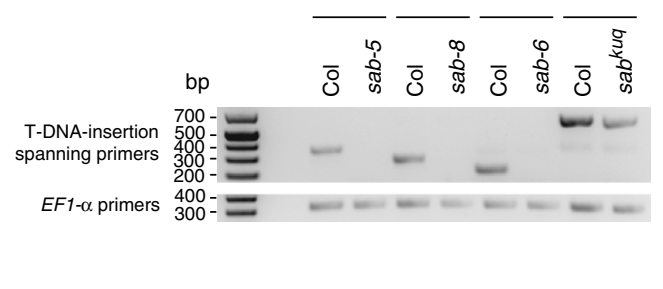

g

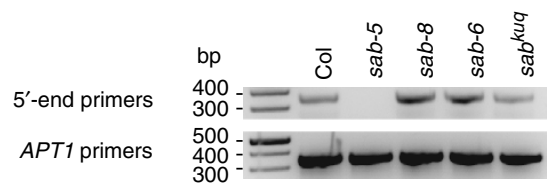

h
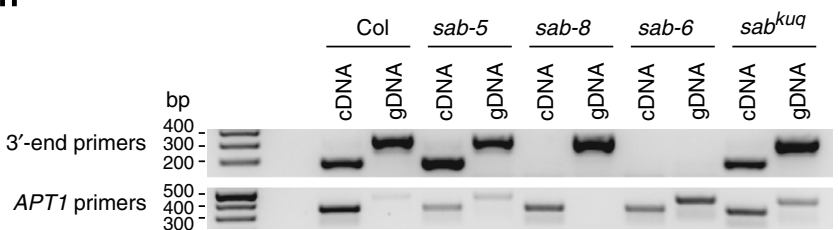

Figure 3 | Phenotypic and RT-PCR analyses of additional SAB T-DNA-mutant alleles. (a) Five-week-old plants of Col, sab-7, sab ${ }^{k u q}$ and $s a b-5$. Note, sab-5 is indistinguishable from sab ${ }^{k u q}$ while sab-7 is less affected. (b) Quantitative analysis of trichoblast length in Col and $S A B$-mutant alleles. Average cell length of $n=150$ trichoblasts from 30 roots per genotype. Data are means \pm s.d. from $n=3$ independent experiments. Statistical differences determined by twotailed, unpaired $t$-test with equal variance and significance level $P<0.05$ are indicated as ${ }^{\star} P<0.05$ and ${ }^{\star \star} P<0.01 ; n=3$. All sab alleles differ significantly from wild type but do not differ from each other $(P>0.05)$. (c-e) Quantitative analysis of planar polarity defects in (c) sab-5, (d) sab-6 and $(\mathbf{e})$ sab-7 compared with $\mathrm{Col}$ is displayed as relative root hair position similar to Fig. 1c. ${ }^{\star \star} P=0.000 \mathrm{Col}$ versus sab-5, sab-6 or sab-7 by K-S test, $n=150$ cells from 30 roots per genotype. (f-h) Semiquantitative RT-PCR analysis of wild-type Col and sab-mutant seedlings. SAB transcript was amplified with (f) primers spanning the T-DNA insertion (or single-nucleotide deletion) and primers located close to (g) the $5^{\prime}$ - or (h) the $3^{\prime}$-end of $S A B$ coding sequence. Primers close to $3^{\prime}$-end were also employed on genomic DNA (gDNA) extracted from the same plant material used for cDNA (cDNA) synthesis. Partial SAB transcripts were detected in all T-DNA alleles. EF1- $\alpha$ and APT1 were utilized as controls. Scale bar, $10 \mathrm{~mm}$.

PROTEIN (H2B-YFP) ${ }^{34}$, respectively, revealed that in comparison with wild type (Fig. 6c), sab-5-mutant cells displayed misorientation of the preprophase band (PPB; Fig. 6d). This orientation defect was followed by similarly misaligned spindle and phragmoplast orientation throughout mitosis and cytokinesis, whereas the overall timing of mitosis and cytokinesis was indistinguishable from wild-type root cells (Fig. 6d; Supplementary Movies 1 and 2). Similar results were obtained in immunolocalization studies demonstrating that PPB, spindle and phragmoplast orientation were defective in epidermal

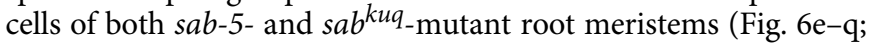
Supplementary Fig. S3f-h). PPB, spindle and phragmoplast orientation in the sab-5;clasp-1 double mutant did not statistically differ from the $s a b-5$ single mutant, but were significantly more strongly affected than in the clasp-1 single mutant (Fig. 6o-q). Thus, $S A B$ function is required for cell division plane orientation already before or at the level of PPB orientation and $s a b$ shows epistasis over clasp during this process. Further, in comparison with wild type (Fig. 6r), the orientation of functional GFP-CLASP ${ }^{21}$ on $s a b-5$ mutant PPBs was affected and coincided with the misoriented alignment of PPB microtubules
(Fig. 6s,t). These results suggested that $S A B$ function is required for orientation of CLASP-guided cortical microtubules and stabilizes correct placement of PPB microtubules in the future plane of cell division.

$S A B$ acts on cortical microtubule arrays. We next analysed potential effects of SAB on cortical microtubule orientation in late meristematic epidermal cells at the transition to cell elongation, in rapidly expanding cells, and during later stages of hair cell differentiation. Strikingly, in contrast to the transversal orientation of mCherry-TUA5-marked microtubules observed in both late meristematic cells (Supplementary Fig. S4a) and in rapidly elongating wild-type cells (Fig. 7a), sab-5-mutant cells of the same size displayed a more random orientation of cortical microtubules than wild-type cells in both cases (Supplementary Fig. S4b,c; Fig. $7 b, c)$. We further monitored the directionality of microtubule growth using the microtubule plus end-tracking protein (+ TIP) AtEB1a-GFP 35 in combination with mCherry-TUA5. This revealed bidirectional movement of transversally oriented cortical microtubules in elongating wild-type cells and of the 
a

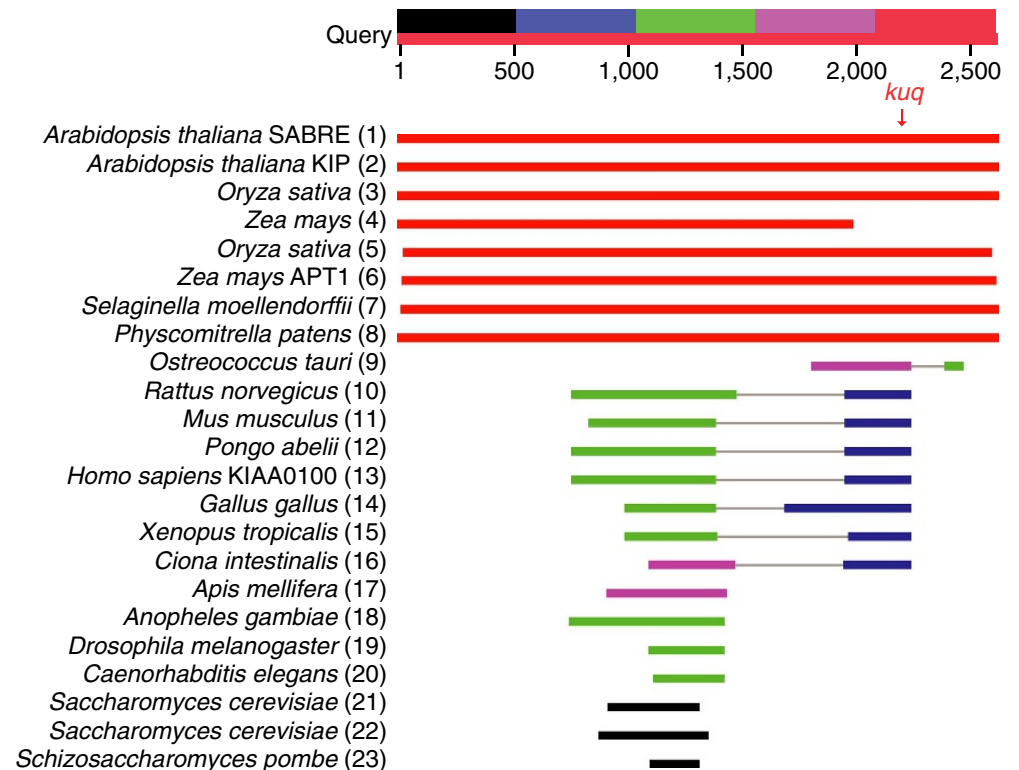

b

b Sacharomyces cerevisiae (21) NP 0154421

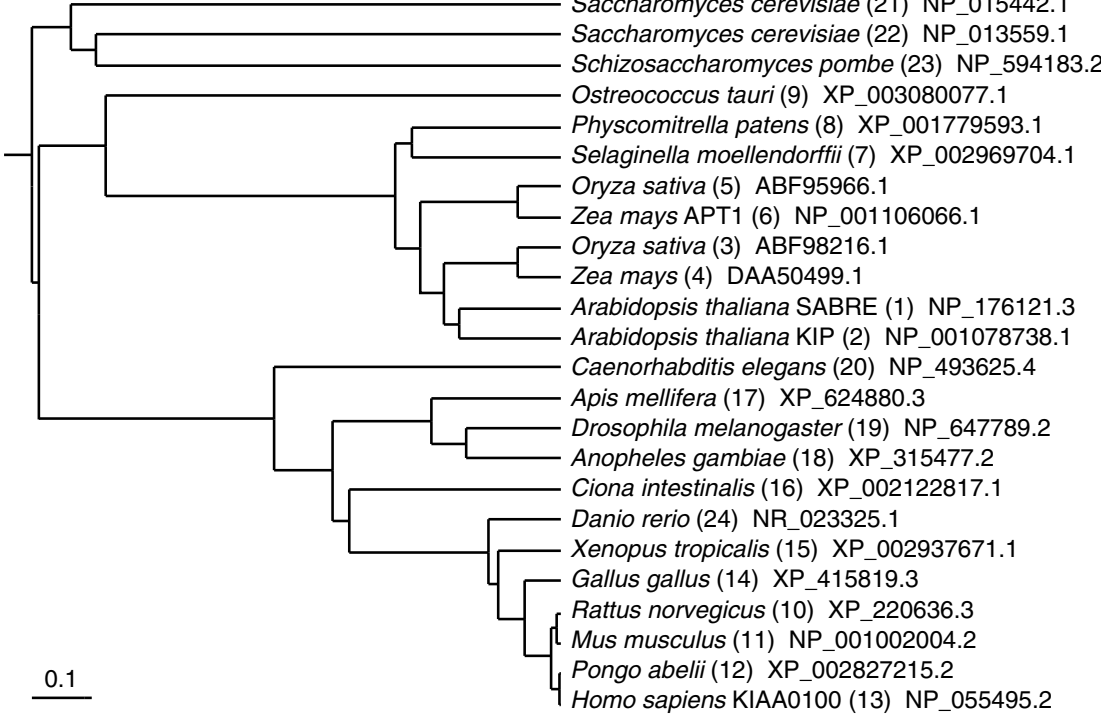

Figure 4 | SAB-related proteins in diverse eukaryotes. (a) Blastp search for proteins homologous to SAB and alignment of SAB-like protein sequences from selected organisms. Note, sequence similarity with proteins from more distantly related species is restricted to two distinct regions of about 600 and 300 amino acids. Red arrow, position of premature stop codon introduced by kuq mutation. Numbers in parentheses refer to sequences in $\mathbf{b}$. Red, alignments with highest sequence identity; black, lowest sequence identity. (b) Phylogenetic tree based on sequences of SAB-related proteins in selected species, showing evolutionary conservation of $\mathrm{SAB}$ across a wide variety of eukaryotic organisms. NCBI reference sequence or GenBank accession number for each protein are indicated next to the respective species.

more randomly oriented microtubules in sab-5-mutant epidermal cells (Fig. 7a,b; Supplementary Movies 3 and 4). Intriguingly, elongating trichoblasts just before hair initiation displayed a reoriented, bipolar arrangement of longitudinally growing microtubules (Fig. 7d; Supplementary Movie 5), where the majority of microtubule plus ends grew with apical directionality at apical ends of cells (Fig. 7e) and with basal directionality at basal ends of cells (Fig. 7f). This bipolarity of microtubule growth resembled the one recently described for elongating hypocotyl cells ${ }^{36}$. The overall longitudinal orientation and bipolarity of microtubule growth was less clearly axially aligned in sab-5-mutant cells (Fig. 7g; Supplementary Movie 6). In addition, mCherry-TUA5 co-visualized with the root hair initiation site marker PIP5K3-YFP ${ }^{37}$ revealed an accumulation of radially reorganized cortical microtubules at the root hair initiation site in $45 \%$ of the cells observed (32/71; Fig. 7h-l; Supplementary Fig. S4d,e), whereas 55\% did not harbour such an array (Supplementary Fig. S4f,g). The majority of cells (94\%, 67/ 71) at this differentiation stage showed longitudinally oriented microtubules interspersed in diffuse, cytosolic mCherry-TUA5 fluorescence indicative of microtubule depolymerization and reorganization in these cells (Fig. 7i; Supplementary Fig. S4d-g). In comparison, only a minority of cells $(6 \%, 4 / 71)$ still revealed transversal microtubule orientation, when hair initiation sites were already marked by PIP5K3-YFP. Although we did occasionally observe reorganized cortical microtubules at PIP5K3-YFP-marked hair initiation sites in sab-5-mutant cells (Fig. $7 \mathrm{~m}-\mathrm{o})$, none of these cells $(0 \%, 0 / 50)$ displayed one unique 
a

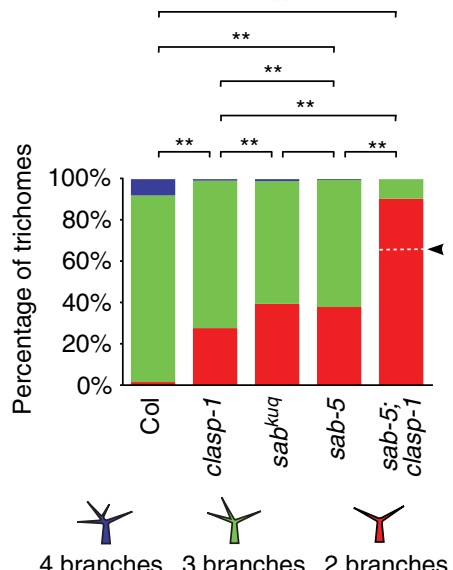

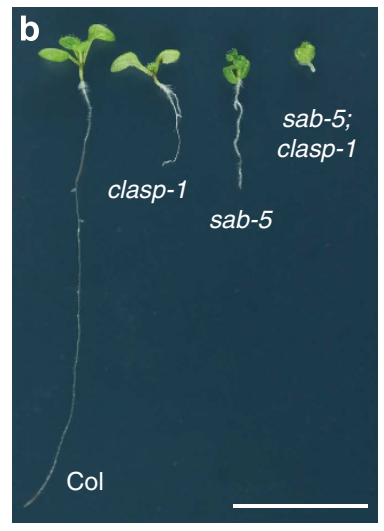
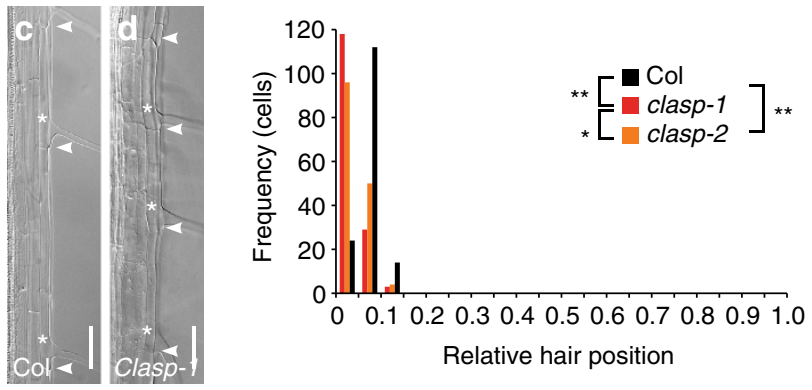

k

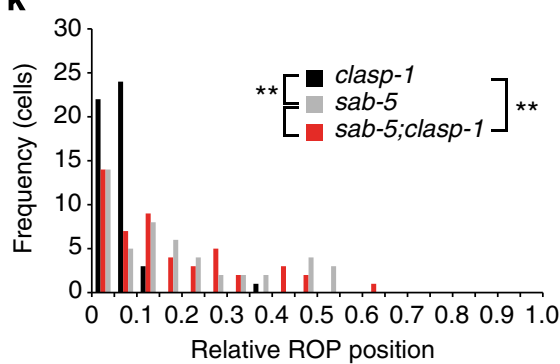

Figure 5 | SAB-CLASP interaction mediates planar polarity upstream of ROP. (a-k) Genetic action of sab and interaction with clasp during (a) leaf trichome branching, (b) seedling development and (c-k) planar root epidermal polarity establishment. (a) Percentage of 2-branched (red), 3-branched (green) and 4-branched (blue) trichomes in Col (total trichome number: $n=545)$, clasp-1 ( $n=537$ ), kuq-mutant allele of $S A B$ (sab ${ }^{k u q} ; n=409$ ), sab-5 $(n=498)$ and sab-5;clasp-1 $(n=431)$ obtained from 10-16 leaves per genotype. Note, significant differences between all genotypes except for sab-5 and sab ${ }^{k u q}(P=0.51)$; the percentage of 2-branched trichomes in sab-5;clasp-1 highly significantly differed from the sum of the respective single-mutant values (dotted line, arrowhead; $P<0.001$ ). Significances were determined by Fisher's exact test with significance level $P<0.05$ and are indicated as ${ }^{\star} P<0.01$. Supplementary Table S1 shows exact $P$-values. (b) Ten-day-old Col, clasp-1, sab-5 and sab-5;clasp-1 seedlings. (c,d) Root hair position on epidermal cells of 5-day-old (c) Col and (d) clasp-1 seedlings. (e) Quantitative analysis of planar polarity in Col, clasp-1 and clasp-2 is displayed as relative root hair position as in Fig. 1c. ( ${ }^{\star \star} P=0.000$ Col versus clasp $-1 ;{ }^{\star \star} P=0.000$ Col versus clasp-2 by K-S test; $n=150$ cells per genotype). (f,g, $\left., \mathbf{i}, \mathbf{j}\right)$ Planar polarity phenotypes revealed by anti-ROP immunolabelling (red), DAPI (blue) overlaid with brightfield image of epidermal cells from (f) Col, (g) clasp-1, (i) sab-5 and (j) sab5; clasp-1. (h,k) Quantitative analysis of polar ROP protein patch positions as in Fig. 1g. (h) Col, clasp-1, clasp-2, (k) clasp-1, sab-5 and sab-5;clasp-1 ${ }^{\star \star} P=0.004 \mathrm{Col}$ versus clasp $-1 ;{ }^{\star \star} P=0.000$ Col versus clasp- $2 ; P=0.241$ clasp -1 versus clasp $-2 ;{ }^{\star \star} P=0.000$ clasp -1 versus sab-5; ${ }^{\star \star} P=0.000$ clasp -1 versus sab-5;clasp-1; $P=0.841$ sab-5 versus sab-5; clasp- 1 by K-S test, $n=50$ cells from 8 to 22 roots per genotype). Arrowheads, apical and basal ends of cells in $\mathbf{c}, \mathbf{d}, \mathbf{f}, \mathbf{g}, \mathbf{i}$ and $\mathbf{j}$. Asterisks indicate sites of hair formation in $\mathbf{c}$ and $\mathbf{d}$. Scale bars, $10 \mathrm{~mm}(\mathbf{b}), 50 \mu \mathrm{m}(\mathbf{c}, \mathbf{d}), 10 \mu \mathrm{m}(\mathbf{f}, \mathbf{g}, \mathbf{i}, \mathbf{j})$.

radial rearrangement that could be unambiguously identified as preceding a hair initiation site comparable to the situation in wild type (Fig. $7 \mathrm{~m}-\mathrm{o}$; Supplementary Fig. S4h-k). Hence, because of the irregular cortical microtubule arrangement in sab-5, microtubule organization did not allow for prediction of the positions of hair initiation sites. However, PIP5K3-YFP-marked hair initiation sites occurred in ectopic basal-most, or more apical positions along sab-5-mutant hair cells (Fig. 7m-p; Supplementary Fig. S4h-k), when compared with the wild type (Fig. 7h-j,p; Supplementary Fig. S4d-g). These findings suggest that $S A B$ activity is required for cortical microtubule reorganization at correct sites before hair outgrowth. Thus, $S A B$ function mediates transversal microtubule alignment in elongating cells and polar placement of domains of reorganizing microtubules in cells initiating hair formation.

SAB subcellular localization. We next determined the subcellular localization of the predicted full-length $290 \mathrm{kDa} \mathrm{SAB}$ protein encoded by a messenger RNA of low abundance ${ }^{27,28}$. To this end, we employed recombineering ${ }^{38}$ to insert three consecutive copies of the cDNA encoding for the bright fluorescent protein Ypet (3xYpet) at the $5^{\prime}$ - or at the $3^{\prime}$-end of the genomic coding sequence of $S A B$. Both constructs rescued the overall $s a b-5$ growth defect and all phenotypes investigated, including cell division plane orientation and planar polarity of root hair initiation (Fig. $8 \mathrm{a}-\mathrm{g}$ ). The planar polarity phenotype of $s a b-5$ was fully rescued by SAB-3xYpet and largely, albeit not completely, by 3xYpet-SAB (Fig. 8f,g). Both constructs were expressed at low levels throughout the root tip and displayed indistinguishable subcellular distribution (Figs $8 \mathrm{~h}-\mathrm{l}$ and $9 \mathrm{a}, \mathrm{b}$ ). These results suggested a likely orientation of both the $\mathrm{N}$ - and the C-terminus towards the cytosol because fluorescence of cell walloriented GFP and YFP protein tags has been reported to be quenched by the low cell wall $\mathrm{pH}$ (refs 39,40). 3xYpet-SAB and $\mathrm{SAB}-3 \mathrm{xY}$ pet localized in patches at the cell periphery, suggesting localization to the plasma membrane or cell wall and to endomembrane compartments (Figs 8k,l and Fig. 9a,b). Plasmolysis experiments in the presence of a cell wall marker revealed retraction of $S A B-3 x Y$ pet from the cell wall (Fig. 9c,d). Strikingly, meristematic cells showed clear enrichment of 

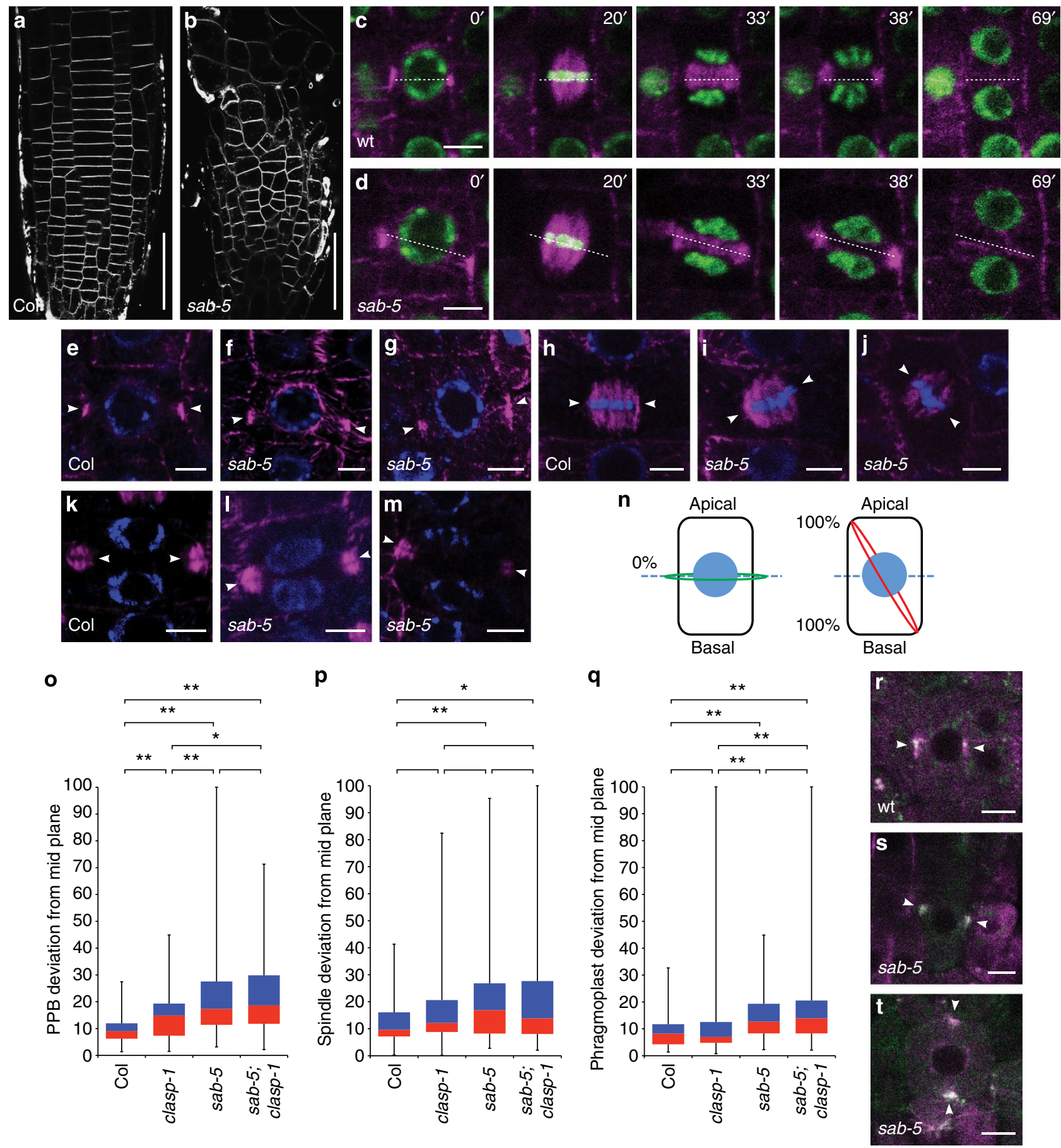

Figure 6 | sab is epistatic to clasp at mediating plane orientation. (a,b) Cell division orientation in root meristematic cells of 5-day-old (a) Col and (b) sab-5 seedlings. Cells are outlined by FM4-64 fluorescence. (c,d) Live imaging of microtubules (mCherry-TUA5, magenta) and DNA (H2B-YFP, green) during cell division in meristems of 5-day-old seedlings. (c) Wild-type (wt; SAB/SAB or SAB/sab-5), (d) sab-5 homozygote (sab-5) cells. Number of cell division experiments analysed: wt, $n=8 ; s a b-5, n=7$. Time points from preprophase band-(PPB)-marked prophase $\left(0^{\prime}\right)$ until the end of cytokinesis $\left(69^{\prime}\right)$ are indicated. Dotted lines, mitotic microtubule array or cell division orientation. (e-m) Mitotic microtubule array orientation in root meristematic cells are revealed by anti- $\alpha$-tubulin immunolocalization (magenta). DAPI-labelled DNA, blue. (e-g) PPB in (e) wild type Col and (f,g) sab-5. (h-j) Metaphase plate in (h) Col and $(\mathbf{i}, \mathbf{j})$ sab-5. ( $\mathbf{k}-\mathbf{m})$ Late phragmoplast in $(\mathbf{k})$ Col and $(\mathbf{I}, \mathbf{m})$ sab-5. Angles of microtubule array orientation were obtained from $n=60$ cells per mitotic stage, per genotype Col, clasp-1, sab-5 and sab-5;clasp-1. Images for clasp-1 and sab-5;clasp-1 are not shown. (e-m) Arrowheads, plane of mitotic microtubule array orientation. (n) Microtubule array deviation from midplane are indicated as $0 \%$ (left) and 100\% (right). (o-q) Quantitative, statistical analyses of anti- $\boldsymbol{\alpha}$-tubulin immunolocalization. (o) PPB, (p) metaphase plate, (q) late phragmoplast orientation in Col, clasp-1, sab-5 and sab-5;clasp-1. (o-q) Box-and-whiskers plots for $n=60$ cells per mitotic stage, per genotype. Whiskers, maximum and minimum values of population. Blue boxes, $25 \%$ of values above median. Red boxes, $25 \%$ of values below median. Statistical differences between populations determined by K-S test are indicated as ${ }^{\star} P<0.05$ and ${ }^{\star \star} P<0.01$ (significance level $P<0.05$ ). For exact $P$-values, see Supplementary Table S2. Note, sab-5; clasp-1 does not significantly differ from sab-5 in $\mathbf{0}$-q, but significantly differs from Col and clasp-1 in $\mathbf{o}$ and $\mathbf{q}$. (r-t) Orientation (arrowheads) and co-localization (white) of GFP-CLASP (green) with mCherryTUA5 (magenta) at PPBs of (r) wild-type (wt; SAB/SAB or SAB/sab-5) and (s,t) sab-5 homozygotes (sab-5). (s,t) Note, aberrant PPB orientation in sab-5. Scale bars, $50 \mu \mathrm{m}(\mathbf{a}, \mathbf{b}), 5 \mu \mathrm{m}(\mathbf{c}-\mathbf{m}, \mathbf{r}-\mathbf{t})$. 

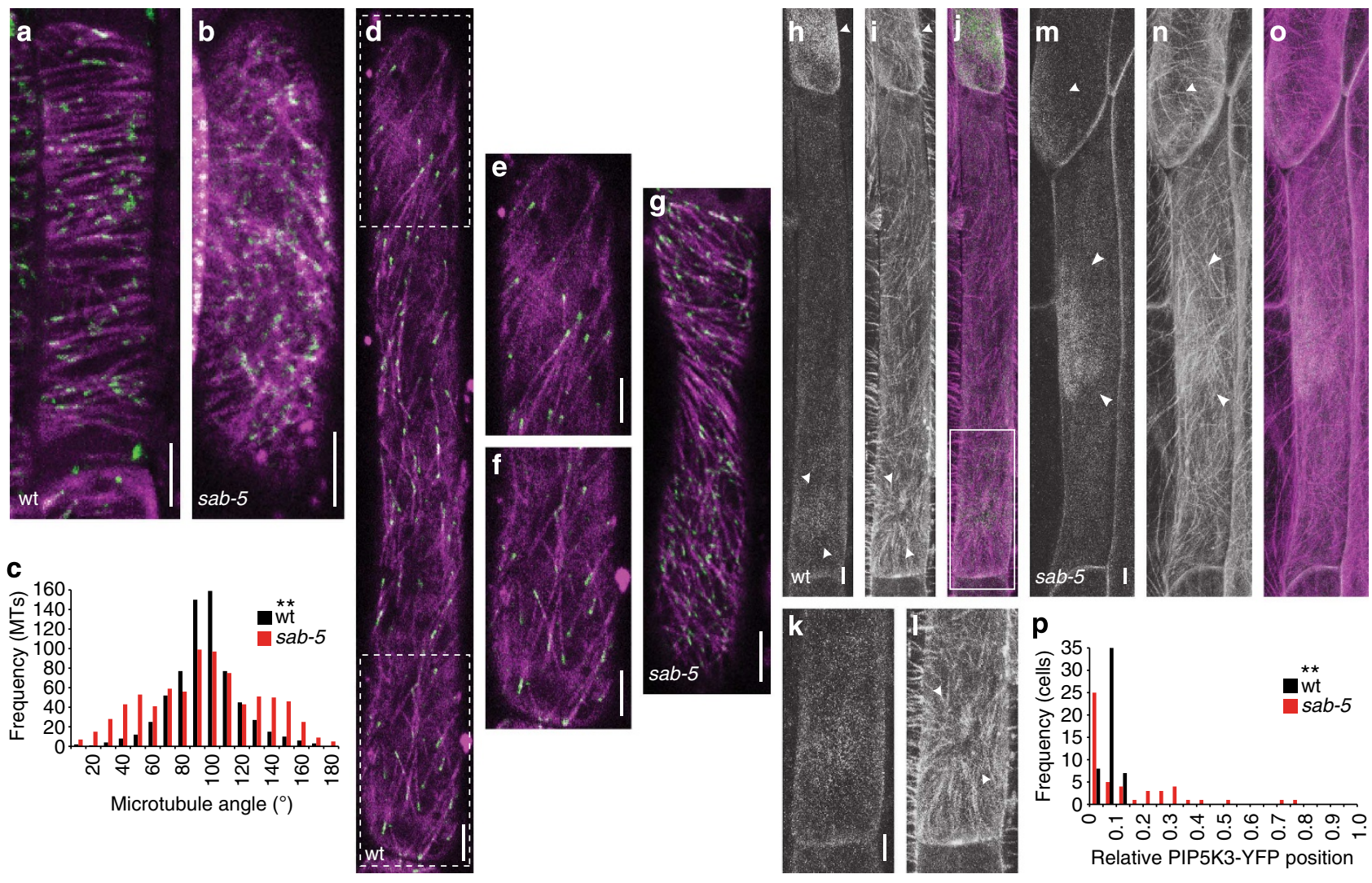

Figure 7 | SAB is required for cortical microtubule orientation during cell elongation and planar polarity. (a,b) Live imaging of cortical microtubule arrays in early elongation stage root hair cells co-expressing mCherry-TUA5 (magenta) and the plus end-tracking protein AtEB1a-GFP (green). Note, (a) transversal orientation in wild type (wt; $S A B / S A B$ or $S A B / s a b-5$ ), versus (b) more random orientation in sab-5 homozygote (sab-5). (c) Quantitative analysis of microtubule angles relative to the apical-basal cell axis in hair cells of $26-36 \mu \mathrm{m}$ length from phenotypically wt and sab-5 seedlings. Statistical analysis reveals highly significant difference between wt and $s a b-5$ ( ${ }^{\star \star} P=0.000$ by K-S test, $n=673$ microtubules analysed for wt and $n=802$ for sab-5, from 28 and 20 cells). For similar results on late meristematic cells of 13-18 $\mu$ m length, see Supplementary Fig. S4a-c. (d-g) Live imaging of cortical microtubule arrays in later elongation stage root hair cells co-expressing mCherry-TUA5 (magenta) and the plus end-tracking protein AtEB1a-GFP (green). (d) Bipolar axial orientation of microtubule growth in wt cell just before first hair formation. (e,f) Magnifications of regions marked in d, showing plus ends of microtubules preferentially growing towards (e) apical and (f) basal ends of cell. ( $\mathbf{g}$ ) Perturbed axial alignment and growth of microtubules in sab-5-mutant cell. (h-o) Live co-imaging of mCherry-TUA5-labelled microtubules with hair initiation site marker PIP5K3-YFP. (h) PIP5K3-YFP, (i) mCherry-TUA5, (j) merge of PIP5K3-YFP (green) and mCherry-TUA5 (magenta) in wt cells. (k,l) Magnifications of inset in $\mathbf{j}$, showing (k) PIP5K3-YFP and (I) mCherry-TUA5 at hair initiation site. (m) PIP5K3-YFP, (n) mCherry-TUA5, (o) merge of PIP5K3-YFP (green) and mCherry-TUA5 (magenta) in sab5. Arrowheads, microtubule (re-)organization at (i) close-to-basal site in wt versus (n) basal-most or apically shifted sites in sab-5. (p) Quantitative analysis of polar PIP5K3-YFP patch position, marking site of root hair initiation. Number of cells in classes (frequency) showing PIP5K3-YFP position between basal ( 0 ) and apical (1) ends of epidermal cells is shown ( ${ }^{\star \star} P=0.000$ wt versus sab- 5 by K-S test, $n=50$ cells per genotype from 39 wt and 41 sab-5 roots). For additional microtubule visualization, see Supplementary Fig. S4d-k. Scale bars, $5 \mu \mathrm{m}$.

SAB-3xYpet at domains of apical and basal plasma membranes that had just started to retract from the cell wall at the onset of plasmolysis (Fig. 9c). Similarly, transversal, apical-basal membranes of non-plasmolysed cells showed an enrichment of SAB-3xYpet (Fig. 9a) and 3xYpet-SAB co-localization with the plasma membrane marker NSPN12-mCherry/WAVE131R (ref. 41; Fig. 9e). Co-visualization of SAB-3xYpet with endoplasmic reticulum (ER; Fig. 9f), Golgi (Fig. 9g) and trans-Golgi network (TGN) (Fig. 9h) markers ${ }^{41,42}$ revealed that the endomembrane compartments containing 3xYpet-SAB/SAB3xYpet did neither represent ER, Golgi nor TGN (Fig. 9f-h). Our findings do not support the previously posed hypotheses that full-length SAB-like proteins may be Golgi-localized or secreted at steady state, respectively ${ }^{28,30}$. Rather, our results provide in vivo evidence corroborating the identification of $\mathrm{SAB}$ peptides from plasma membrane preparations by proteomic approaches ${ }^{43}$. Further, SAB-3xYpet accumulated in small patches at the nuclear envelope where it co-labelled with microtubules marked by
mCherry-TUA5 during mitotic prophase (Fig. 9i). At the cell cortex, SAB-3xYpet did not precisely co-localize with PPB microtubules but consistently closely associated with them in the majority of prophase cells analysed $(91 \%, 84 / 92)$ (Fig. 9i, arrowheads; Supplementary Fig. S5a-f). Strikingly, SAB-3xYpet was also found in patches along mitotic spindle microtubules (Fig. 9j,k), located to cell plate membranes from early to late stages of cell plate formation (Fig. 9l-n), and subsequently remained at domains of apical and basal membranes of dividing cells (Fig. 9e,n). These findings were confirmed in co-labelling studies with rhodamine phalloidin for F-actin staining in aldehyde-fixed cells (Fig. 9o-t) and by co-labelling of the KNOLLE cell plate syntaxin with SAB-3xYpet at the cell plate (Fig. 9u). In elongating epidermal cells, SAB-3xYpet distributed more evenly in endomembrane compartments of unknown identity throughout the cytosol (Fig. 9b). In more differentiated cells, where SAB-3xYpet expression was very low and just detectable above background, SAB-3xYpet compartments were 

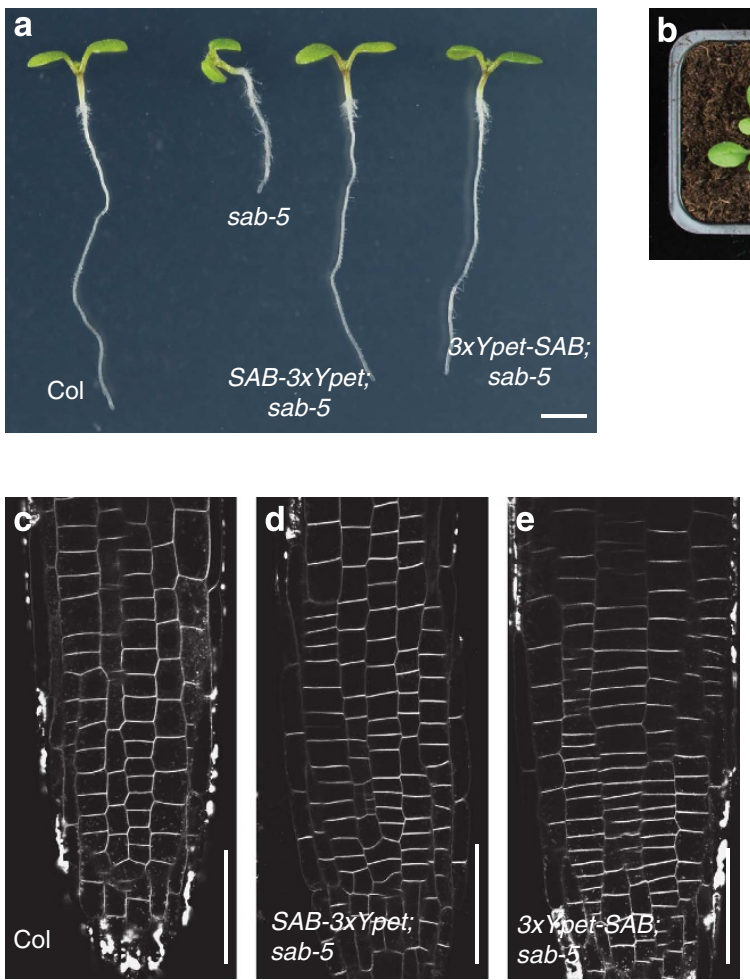

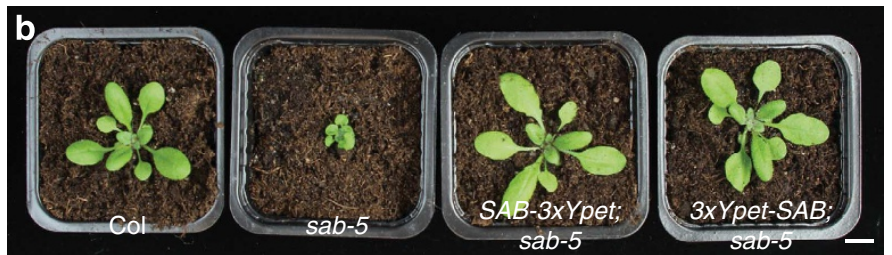

f

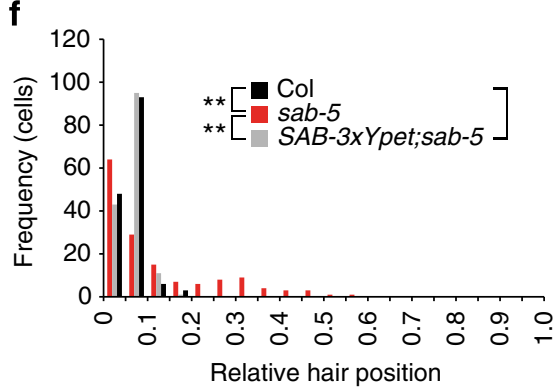

g

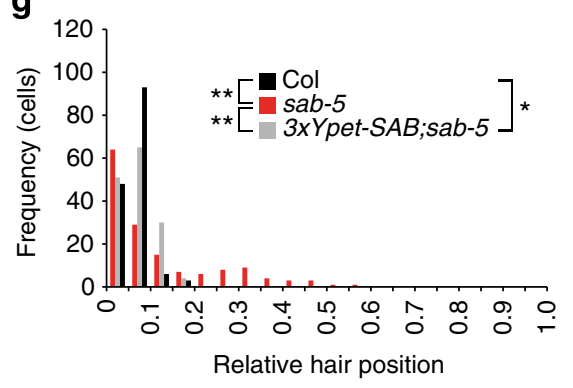

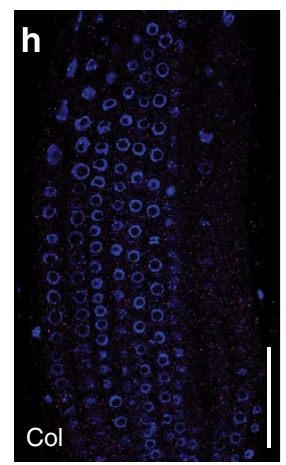
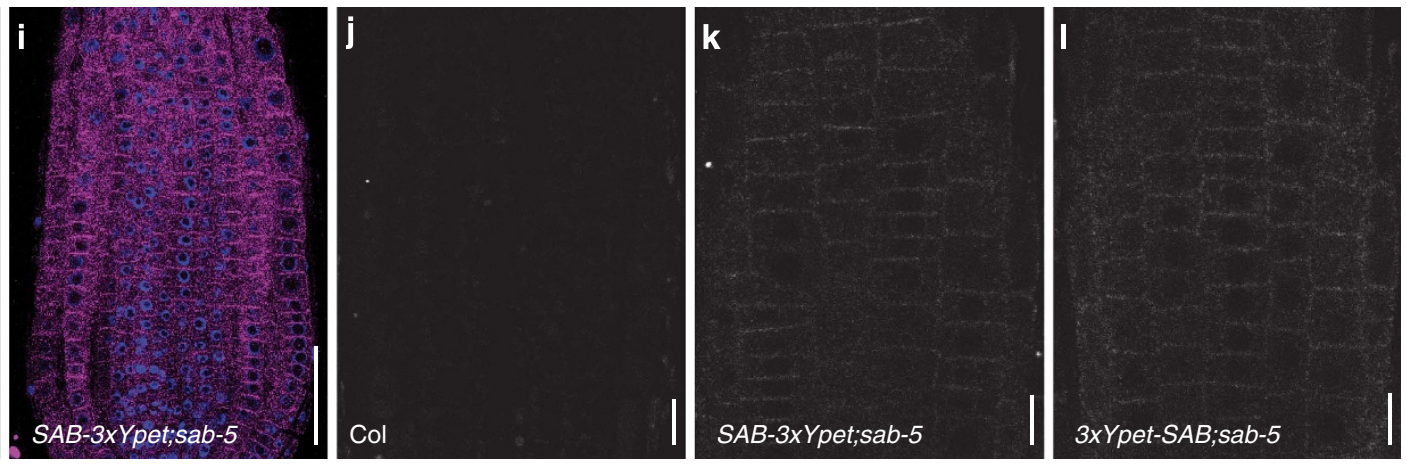

Figure 8 | SAB-3xYpet and 3xYpet-SAB rescue sab-5 phenotypes and display identical localization. (a) Five-day-old seedlings, (b) 21-day-old plants of Col, sab-5, SAB-3xYpet;sab-5 and 3xYpet-SAB;sab-5. Note, rescue of sab-5 growth defects. (c-e) Cell division orientation in root meristem of 5-day-old (c) Col, (d) $S A B-3 x Y$ pet;sab-5 and (e) 3xYpet-SAB;sab-5 seedlings. Cells outlined by FM4-64 fluorescence. (f,g) Quantitative analysis of planar polarity in (f) $S A B-3 x$ Ypet;sab-5 and (g) $3 x Y$ pet-SAB;sab-5 compared with Col and sab-5 is displayed as relative root hair position similar to Fig. 1c. ${ }^{\star \star} P=0.000 \mathrm{Col}$ versus sab-5; $P=0.422$ Col versus $S A B-3 x Y$ pet;sab-5; ${ }^{\star \star} P=0.000$ sab-5 versus $S A B-3 x Y$ pet;sab-5; $P=0.019$ Col versus $3 x Y$ pet-SAB;sab-5; ${ }^{\star \star} P=0.000$ sab-5 versus $3 \times Y$ pet-SAB;sab-5 by K-S test, $n=150$ cells from 30 roots per genotype. Note, (f) full rescue of sab-5 planar polarity defects by $S A B-3 \times Y p e t$ and (g) partial rescue by $3 x$ Ppet-SAB. (h,i) Expression of SAB-3xYpet throughout the root meristem was detected by immunolabelling with anti-GFP antibody (magenta), DAPI (blue), of (h) Col and (i) SAB-3xYpet;sab-5 seedlings. Images are transversal sections showing SAB-3xYpet signal in all cell layers. (j-I) Live imaging of (j) Col, (k) SAB-3xYpet;sab-5 and (I) 3xYpet-SAB;sab-5 seedlings showing indistinguishable subcellular localization of SAB-3xYpet and 3xYpet-SAB in root meristematic epidermal cells. Scale bars, $2 \mathrm{~mm}(\mathbf{a}), 10 \mathrm{~mm}(\mathbf{b}), 50 \mu \mathrm{m}(\mathbf{c}-\mathbf{e}, \mathbf{h}, \mathbf{i}), 10 \mu \mathrm{m}(\mathbf{j}-\mathbf{I})$.

present, although not much enriched, at sites of root hair initiation before morphological outgrowth (Fig. 9v,w). Taken together, we show that SAB localizes to plasma membrane domains, preferentially but not exclusively to apical and basal plasma membranes of meristematic cells, at the nuclear periphery, flanking the PPB at the onset of mitosis, to mitotic spindles, to cell plate membranes as well as to as yet unidentified endomembrane compartments. Thus, SAB is present in most root tip cells including dividing, elongating and differentiating epidermal cells, consistent with its functions in epidermal cells reported here.

\section{Discussion}

Our study unravels new roles for the large $\mathrm{SAB}$ protein related to uncharacterized proteins found throughout eukaryotes. We report important functions for $S A B$ in $\mathrm{PPB}$, spindle, phragmoplast and cell division plane orientation, cortical microtubule orientation and planar polarity establishment. Moreover, we uncover biological functions of $S A B$ in cell and planar polarity that it shares with CLASP. A role for CLASP function during microtubule-dependent PIN2 polarity establishment in cortical cells and PIN2 abundance modulated by endocytosis has recently attracted attention ${ }^{18,19}$. Although $S A B$ and CLASP do not 

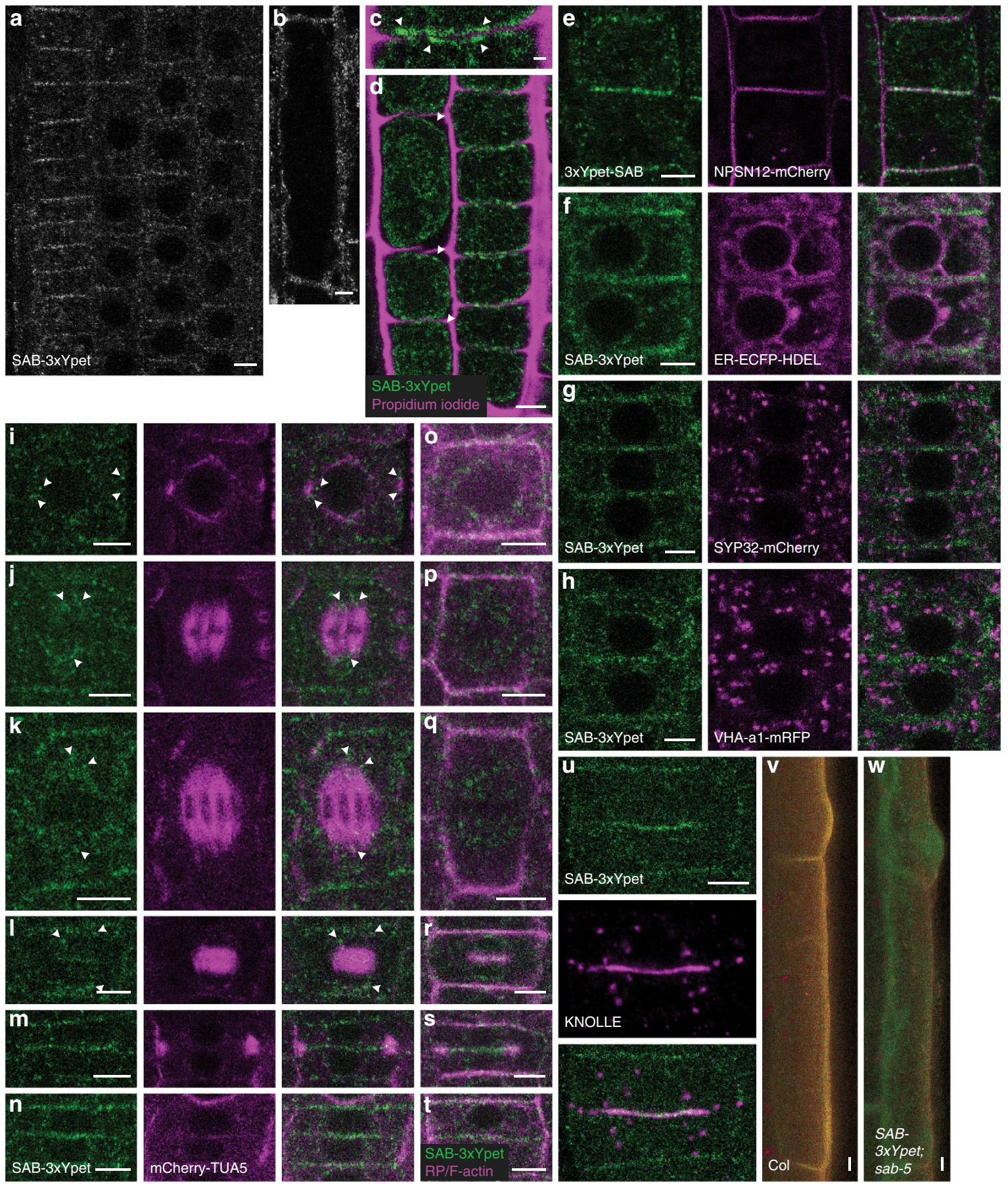

\section{g}
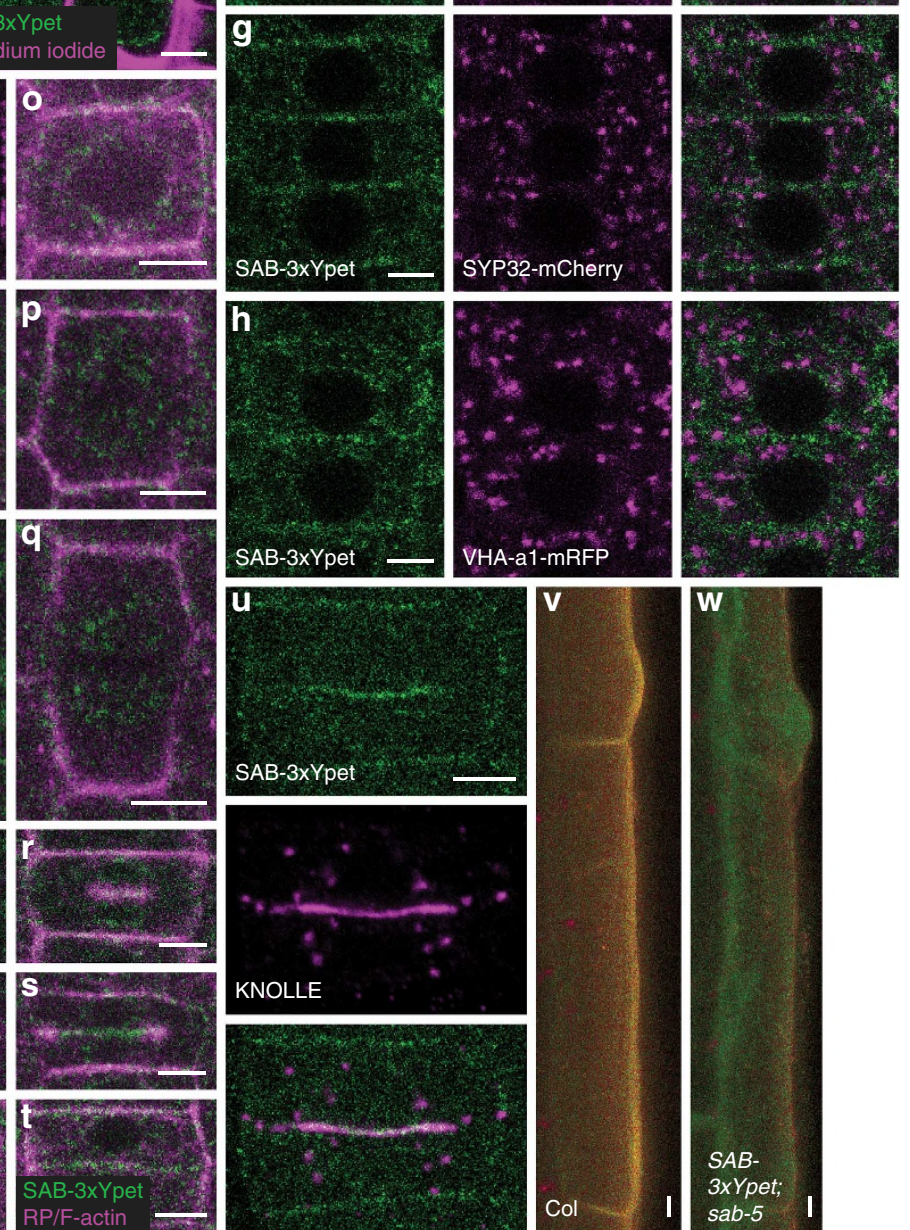

Figure 9 | SAB subcellular localization. (a-h,v,w) Live imaging of functional SAB-3xYpet or 3xYpet-SAB in root epidermal cells of 5-day-old seedlings. (a,b) SAB-3xYpet in sab-5 background detected in (a) meristematic cells and (b) elongating cells. (c,d) Onset of plasmolysis in 5-day-old, SAB-3xYpet;sab-5 seedling root co-labelled for SAB-3xYpet (green) and cell wall stain propidium iodide (magenta). Note, (c) apical and basal membrane domain labelling (arrowheads) and (d) separation of SAB-3xYpet from cell wall (arrowheads). (e-h) Meristematic root epidermal cells. (e) 3xYpet-SAB (green) co-localizes with plasma membrane marker NPSN12-mCherry/WAVE131R (magenta). (f-h) SAB-3xYpet (green) co-detected with (f) ER marker ER-ECFP-HDEL

(magenta), (g) Golgi marker SYP32-mCherry/WAVE22R (magenta) and (h) TGN marker VHA-a1-mRFP (magenta). Merged channels to the right in e-h. Note, (f) little co-localization and ( $\mathbf{g}, \mathbf{h})$ no co-localization when compared with $\mathbf{e}$. (i-t) SAB-3xYpet (green) (i-n) co-expressed with mCherry-TUA5 (magenta) and (o-t) co-labelled with rhodamine phalloidin (magenta) for F-actin visualization (RP/F-actin) in paraformaldehyde-fixed roots. (i) Prophase. SAB-3xYpet and mCherry-TUA5 fluorescence around the nuclear envelope. Note, association of SAB-3xYpet (arrowheads) with PPB but no direct colocalization (see also Supplementary Fig. S5). (o) Late prophase. SAB-3xYpet and RP/F-actin. (j,k) SAB-3xYpet localization at mitotic spindles preferentially towards the periphery (arrowheads) in (j,p) metaphase and $(\mathbf{k}, \mathbf{q})$ anaphase. (I,r) Late telophase. (I) SAB-3xYpet in centre of phragmoplast at early cell plate and at remaining spindle microtubules (arrowheads). (r) SAB-3xYpet co-labelling with F-actin at early cell plate. (m,s) Late cytokinesis. SAB-3xYpet at cell plate co-labelling with $(\mathbf{m})$ peripheral phragmoplast microtubules and (s) peripheral phragmoplast F-actin. (n,t) End of cytokinesis. SAB-3xYpet at transversal (apical, basal) plasma membranes. (u) anti-KNOLLE cell plate marker immunofluorescence (magenta) and SAB-3xYpet (green) fluorescence. Channel merge (lower panel) indicates co-localization at cell plate. $(\mathbf{v}, \mathbf{w})$ Elongated epidermal cells undergoing hair initiation and bulge outgrowth, visualized by colour-coded spectral lambda scan between 416 and $686 \mathrm{~nm}$. Ypet-specific fluorescence, green. (v) Wild type Col. (w) Genomic SAB$3 x$ Ypet;sab-5. Images in a-w acquired with LSM780 32-channel gallium arsenide phosphide detector array. Scale bars, $5 \mu \mathrm{m}(\mathbf{a}, \mathbf{b}, \mathbf{d}-\mathbf{x}), 1 \mu \mathrm{m}(\mathbf{c})$. 
appreciably affect apical epidermal PIN2 polarity, we uncover that CLASP mediates establishment of planar polarity of ROP and root hair positioning, and we confirm a previously reported role of CLASP in PPB orientation ${ }^{15,16}$. Intriguingly, however, our results unravel that $s a b$ mutations perturb all investigated processes more strongly than clasp mutations. sab affects PPB, spindle and phragmoplast orientation as well as planar root hair polarity more strongly than clasp, and shows an epistatic interaction with clasp. Synergistic action of $S A B$ and CLASP in controlling root length and trichome branching may reflect additional nonlinear interaction, including potential feedback regulation. Alternatively, synergistic action can be interpreted by interaction of $S A B$ and $C L A S P$ with additional partners during these two processes. However, during PPB, spindle and phragmoplast orientation as well as planar polarity in root epidermal cells, $S A B$ and CLASP act sequentially. Moreover, $S A B$ likely acts upstream of CLASP considering the misorientation of CLASP-labelled PPBs observed in $s a b$, the stronger phenotypes of $s a b$ mutants and the epistatic relationship of $s a b$ over clasp. Hence, our observations mechanistically link $\mathrm{SAB}$ function to CLASP-guided cortical microtubule organization during these processes. We further describe a newly observed bipolar arrangement of longitudinally growing microtubules at later stages of root hair cell elongation. Although striking and similar to the bipolar arrangement recently observed in hypocotyl cells ${ }^{36}$, the functional importance of this dynamic bipolar reorganization, and how it may affect polar site selection during root hair initiation, remains to be determined. Further, we find that $s a b$ is epistatic to clasp during polar placement of ROP protein patches at sites of hair initiation where we observe cortical microtubule reorganization before morphological onset of hair outgrowth. Earlier observations have implicated microtubules in tip growth of different cell types in various organisms ${ }^{14,44-46}$, and radial microtubule alignment has been observed, for example, in tips of moss protonemata $^{44}$ as well as in conifer pollen tubes ${ }^{45}$. In addition, microtubule dynamics are known to have a function in directing the growth of and limiting the number of growth sites in root hairs ${ }^{46,47}$. Our work also points out a role for microtubule reorganization in polarity of root hair cells. However, we report that microtubules are involved earlier than previously described, namely, during selection of the site of root hair initiation. This suggests that the microtubule cytoskeleton acts both in determining planar polarity of hair cell differentiation and in maintaining polar tip growth of root hairs. It remains to be determined how $S A B$ affects microtubule reorganization at the molecular level because the genetic interactions observed between $S A B$ and CLASP may not necessarily reflect direct interaction at the protein level. Yet, SAB-3xYpet localized to the perinuclear region from where microtubules radiate towards the PPB as well as adjacent to the $\mathrm{PPB}$, suggesting that $\mathrm{SAB}$ may be, directly or indirectly, involved in nucleation or guidance of $\mathrm{PPB}$ microtubules. This localization does, however, not match exactly the distribution of CLASP, which directly co-localizes with PPB microtubules at cell edges and binds to microtubule plus ends ${ }^{20,21}$. This suggests that the observed genetic interactions may reflect an indirect or transient interaction within the same pathway. The $\mathrm{SAB}$ protein localizes to spindle microtubules from early prophase, and later on is observed close to the spindle periphery consistent with our findings that $S A B$ is required for spindle and phragmoplast orientation. Further, the accumulation of $\mathrm{SAB}$ at cell plate membranes in the centre of the phragmoplast and on apical and basal plasma membranes opens the door for future studies on the role of $\mathrm{SAB}$ at these sites. Hence, our work paves the way to elucidating the molecular function of $S A B$ in microtubule reorganization during cell division plane orientation, cell and planar polarity formation in plants. It also encourages further studies on the connection between SAB-related proteins and the microtubule cytoskeleton. Future studies may now address what proteins the large membrane protein SAB interacts with at the molecular level and which of the homology regions observed in SAB-like proteins throughout eukaryotes may be required for such interactions. Interestingly, the human genome encodes a single SAB-related predicted 2,235 amino-acid-long protein of unknown function named KIAA0100, which is overexpressed in human cancers and also known as, for example, breast cancer overexpressed 1 or monocytic leukaemiaassociated antigen 22 (refs 48-51). Hence, our findings linking $\mathrm{SAB}$ to CLASP-mediated microtubule organization may provide a stepping stone for functional analysis of SAB-related proteins during eukaryotic development and for dissecting their potential role in human cancer.

\section{Methods}

Plant growth conditions. Medium and growth conditions were as described ${ }^{4}$. In brief, seeds were surface sterilized and stratified at $4{ }^{\circ} \mathrm{C}$ for 3 days before plating on MS plates $(1 \times$ MS medium, $1 \%$ sucrose, $0.8 \%$ plant agar, $1 \mathrm{M}$ morpholinoethanesulphonic acid, pH 5.7). Seedlings were grown vertically at $23^{\circ} \mathrm{C}$ day and $18^{\circ} \mathrm{C}$ night under $16 \mathrm{~h}$ light $/ 8 \mathrm{~h}$ dark photoperiod and subjected to analysis after 5 days.

Plant material and genotyping. Arabidopsis thaliana ecotype Columbia (Col-0) was employed as a wild type and as background for the kuq mutant; the following T-DNA insertion lines from the SALK collection ${ }^{52}$ were obtained from the Nottingham Arabidopsis Stock Centre: sab-5 (stock number N608709), sab-6 (N552995), sab-7 (N623771) and sab-8 (N512897). All above sab alleles, with the exception of $s a b-7$, were unable to self-fertilize and produced 5-10 seeds per silique on pollination with wild-type pollen. Lines were maintained as heterozygotes and molecular genotyping was used to identify plants carrying mutant alleles Other previously described mutants and transgenic lines were provided by the Nottingham Arabidopsis Stock Centre or corresponding authors of the cited publications: pPEN3:PEN3-GFP ${ }^{53}$, p35S:GFP-BASL ${ }^{24}$, clasp-1 (refs 15,16) (N620061), clasp-2 (refs 15,16) (N583034), p35S:mCherry-TUA5 (refs 32,33), p35S:H2B-YFP $3^{34}, p C L A S P: G F P-C L A S P^{21}$, p35S:EB1a-GFP ${ }^{35}$, pGL2:NTF $F^{54}$, pPIP5K3:PIP5K3-YFP ${ }^{37}$, pUBQ10:NSPN12-mCherry/WAVE131R ${ }^{41}$, pUBQ10:SYP32-mCherry/WAVE22R ${ }^{41}$ and VHA-a1-mRFP ${ }^{42}$. Other mutant and transgene combinations were generated in this study. Primers for PCR genotyping of mutants are listed in Supplementary Table S3.

Immunofluorescence and CLSM. Whole-mount immunolabelling of roots was performed as described ${ }^{4}$. Antibody dilutions were the following: rabbit anti-ROP 1:25 (ref. 8); rabbit anti-PIN2 1:400 (ref. 55); mouse anti- $\alpha$-tubulin DM1A 1:200 (Santa Cruz, Sc-32293); rabbit anti-GFP 1:1,000 (Torrey Pines, TP-401); rabbit anti-KNOLLE 1:4,000 (ref. 56); DY649 donkey anti-rabbit 1:800 (Jackson ImmunoResearch) for anti-ROP, anti-PIN2, anti-GFP; DyLight633 donkey antirabbit 1:100 (Agrisera, AS122034) for anti-KNOLLE; TRITC donkey anti-mouse 1:150 (Jackson ImmunoResearch). Donkey secondary antibodies for multilabelling pre-absorbed to crossreacting species were employed. DNA was stained with $4^{\prime}, 6$-diamidino-2-phenylindole (DAPI) $\left(1 \mu \mathrm{g} \mathrm{ml}^{-1}\right.$, Sigma). Imaging of immunolabelled or live roots was carried out using either a Leica TCS SP2 AOBS (Leica) spectral confocal laser scanning microscopy (CLSM) system mounted on an Leica DM IRE2 inverted microscope or a Zeiss LSM780 spectral CLSM system equipped with a 32-channel gallium arsenide phosphide detector array and mounted on a Zeiss Axio observer Z1 inverted microscope. Laser excitation lines, emission filters and acquisition modes for all fluorophore combinations are listed in Supplementary Table S4. Oil-corrected Plan-Apochromat $63 \times$ objectives, numerical aperture 1.4 (HCX PL APO $63.0 \times 1.40$ OI BD UV, Leica; or DIC M27, Zeiss) were used for immunolabelling studies. For live imaging, a water-corrected C-Apochromat $40 \times$ objective, numerical aperture 1.2 (M27, Zeiss) was used. It was not possible to detect $3 \mathrm{xY}$ pet-SAB and SAB-3xYpet fluorescent fusions with a Leica SP2 confocal microscope in live imaging studies because of extremely low intensity of the signal. Hence, all imaging of these lines was carried out with a Zeiss LSM780 microscope. It was however possible to detect SAB-3xYpet and 3xYpetSAB with a Leica SP2 microscope after immunolabelling with anti-GFP antibody (Torrey Pines). Images were processed and analysed using ZEN2010 (Zeiss), ImageJ (http://www.imagej.nih.gov/ij/) or Adobe Photoshop CS4 (Adobe Systems), and assembled in Adobe Illustrator CS3 (Adobe Systems).

Analysis of root hair position and trichoblast length. Quantitative microscopic analysis of root hair position and cell length was performed in three independent experiments as described ${ }^{4}$. Statistical analysis employed non-parametric Kolmogorov-Smirnov (K-S) test and two-tailed $t$-test. 
Quantitative analysis of ROP positioning. Relative ROP position in trichoblasts before hair initiation was determined by immunolocalization as described ${ }^{4}$. Measurements were performed on $n=50$ cells per genotype, from 8 to 22 roots. Images were analysed using Leica Application Suite Advanced Fluorescence software (Leica).

Scanning electron microscopy of leaf trichome orientation. Growth of $s a b$ mutants was significantly slower compared with wild-type plants. To analyse leaves at a similar developmental stage, the third true leaf from 21-day-old sab plants was compared with the third true leaf from 14-day-old wild-type plants that did not obviously differ in trichome orientation from 21-day-old wild-type plants. Leaves were fixed in $2.5 \%$ glutaraldehyde in sodium cacodylate buffer overnight at room temperature. To keep the leaves submerged in the solution, the fixation was carried out in a desiccator with a low under-pressure to take away trapped air. Fixed material was dehydrated in increasing ethanol concentrations, up to pure ethanol, and was thereafter dried from liquid $\mathrm{CO}_{2}$ using standard critical point drying technique (Polaron E3000 CPD apparatus, Quorum Technologies Ltd, Ashford, UK). Dried specimens were attached to specimen holders using adhesive carbon tape and thereafter coated with $\sim 15 \mathrm{~nm}$ gold in an evaporation system. Microscopy was performed in a scanning electron microscope running at standard conditions (Carl Zeiss SMT/Cambridge Instruments 360iXP, Cambridge, UK) and micrographs were recorded at standardized magnifications and locations on the leaves. Trichome orientation was investigated in a rectangle of $2 \mathrm{~mm} \times 1.5 \mathrm{~mm}$ in the centre of the leaf adaxial surface. Angles between the proximal-distal leaf axis and the trichome's first branch were measured on scanning electron microscope images using ImageJ.

Isolation and positional cloning of kuq. The kuq mutant was isolated in a genetic screen of 5-day-old seedlings for aberrant planar polarity of root hair positioning. A population of $\sim 20,000 \mathrm{M}_{2}$ seedlings obtained from $\sim 2,500$ fast-neutronmutagenized $\mathrm{M}_{1}$ plants (Col-0 ecotype, Lehle Seeds) were grown vertically and screened by stereomicroscopy. kuq was characterized by a more random root hair position along epidermal cells, shorter and radially swollen roots and bent cotyledons. The semi-sterile kuq mutant was fertilized with Col-0 pollen and produced five to eight seeds, which were subjected to further analyses. The kuq phenotype segregated at $27 \%(n=496)$ after backcrossing, indicating that it was caused by a single, recessive mutation. $k u q$ was crossed to Landsberg erecta (Ler) to generate a mapping population, and genomic DNA (gDNA) was extracted from 1,298 $\mathrm{F}_{2}$ seedlings displaying the kuq phenotype and used for mapping by positional cloning ${ }^{57}$. SSLP and CAPS molecular markers were generated as described ${ }^{57}$ and by using the Monsanto Arabidopsis Polymorphism Collection (http:// www.arabidopsis.org/browse/Cereon/index.jsp), and the mutation was mapped to a 480-kb interval on chromosome 1 (Fig. 2). Analysis of T-DNA insertion lines in genes within this interval suggested the SABRE (SAB) gene as the strongest candidate, with 9 T-DNA lines whose homozygous seedlings looked indistinguishable from kuq mutants. The entire $\sim 14.5 \mathrm{~kb} S A B$ genomic region was PCR amplified from kuq mutants and sequenced with three times coverage. This revealed a single cytosine deletion in the 17th exon of the $S A B$ gene, at position 6,592 (of 7,824) of the coding sequence. The mutation caused a frame shift that was calculated to introduce a stop codon at position 6,617-6,619, truncating the predicted SAB protein after amino acid 2,205 (of 2,607). A CAPS marker designed over the mutated site physically confirmed the nucleotide deletion and allowed molecular genotyping of kuq mutants (see Supplementary Table S3).

RT-PCR analysis. Total RNA was extracted from roots of 5-day-old seedlings employing the RNeasy Plant Mini Kit (Qiagen) according to manufacturer's instructions. Two biological replicates were analysed for each mutant and the respective wild-type control. RNA was purified with the Ambion DNA-free Kit (Ambion), precipitated with $\mathrm{LiCl}$ and resuspended in diethyl pyrocarbonate-treated $\mathrm{H}_{2} \mathrm{O}$. cDNA was synthesized using iScript cDNA Sythesis Kit (Bio-Rad) and used for semiquantitative RT-PCR analysis with primer pairs spanning the T-DNA insertion (or single-nucleotide deletion), and primer pairs located respectively at the $5^{\prime}$ - and $3^{\prime}$-end of the $S A B$ gene (Supplementary Table $S 3$ for primer sequences). Primer pairs were designed to span introns to identify potential gDNA contaminations. Primers amplifying the ELONGATION FACTOR 1- $\alpha(E F 1-\alpha)$ or ADENINE PHOSPHORIBOSYL TRANSFERASE 1 (APT1) genes were used as controls. Reactions were carried out with 34 cycles for T-DNA insertion spanning primers ( 22 cycles for EF1- $\alpha$ primers) and 35 cycles for primers located close to the $5^{\prime}$ - or $3^{\prime}$ - end of the $S A B$ gene ( 35 and 40 cycles for APT1 primers, respectively).

Analysis of SAB evolutionary conservation. Searches for proteins with similarity to $\mathrm{SAB}$ were performed with Blastp version 2.2.28 + (http://www.blast.ncbi.nlm.nih.gov/blast/Blast.cgi) with default parameters using full SAB sequence (Atlg58250.1) against non-redundant protein sequences database. Alignment of Saccharomyces cerevisiae and Schizosaccharomyces pombe proteins was achieved after second PSI-BLAST iteration. Full sequences of SAB-related proteins from selected species were aligned with MUSCLE version 3.8.31 (http://www.ebi.ac.uk/ Tools/services/web/toolform.ebi?tool=muscle). Output phenogramme generated from second iteration was visualized with Phylodendron Phylogenetic tree printer (http://www.iubio.bio.indiana.edu/treeapp/treeprint-form.html).

Trichome branching analysis. Measurements of trichome branching were performed as described ${ }^{15}$. Third true leaf of 21-day-old plants was observed with a Leica MZ 9.5 stereomicroscope (Leica), and number of branches was counted for all trichomes on adaxial leaf surface. Number of leaves (plants) analysed are as follows: 10 for Col-0, 12 for clasp-1, 16 for $s a b^{k u q}, 16$ for $s a b-5$ and 16 for sab-5;clasp-1.

FM4-64 staining. Seedlings were incubated in $1 \times$ MS medium with $25 \mu$ M FM464 (Molecular Probes) for $5 \mathrm{~min}$ on ice. After two washes with $1 \times$ MS medium on ice, seedlings were mounted on cover slips in $1 \times$ MS medium.

Live imaging of microtubules and nuclei during cell division. Wild-type looking and $s a b-5$ seedlings expressing mCherry-TUA5 (ref. 32) and H2B-YFP ${ }^{34}$ were imaged live. A region of $53.14 \mu \mathrm{m} \times 53.14 \mu \mathrm{m}$ centred on a meristematic cell in prophase (displaying clear PPB) was visualized at intervals of $1 \mathrm{~min}$ throughout cell division. For each time point, $\mathrm{Z}$ stacks of six optical sections at $0.46 \mu \mathrm{m}$ distance were acquired, and the image closest to the centre of the cell was selected. Imaging windows and planes were constantly adjusted to account for movement of roots in $X$-, $Y$ - and $Z$-direction. Images of each time point were aligned using ImageJ Stackreg plugin (http://www.bigwww.epfl.ch/thevenaz/stackreg/) before movie assembly.

Analysis of mitotic figure orientation. Seedlings were labelled with mouse anti$\alpha$-tubulin antibody (Santa Cruz). Z stacks were acquired from meristematic cells in prophase, metaphase and late cytokinesis, and images closest to the centre of the cell were selected. For each cell, length of the longitudinal cell edges and distances between the transversal cell edges and the points where the PPB ring or phragmoplast touched the longitudinal edges were measured. Deviation of PPB, mitotic spindle and phragmoplast orientation from the transversal central axis (midplane) of the cell was indicated by the absolute value derived by the following formula: 100 - (distance between transversal edge and mitotic structure/total length of longitudinal edge $\times 200$ ). A mean of $0 \%$ between the two values calculated for a cell (one corresponding to each side) indicated a structure passing through the midplane, whereas $100 \%$ was one tracing a diagonal of the cell. Higher deviations occurred rarely (2.5\% of 720 cells in total) and were considered as $100 \%$ for further analysis. Box-and-whisker plots were used to visualize deviation from the midplane in the 60 cells examined for each genotype and mitotic stage: for each data set they display median, upper quartile (blue box), lower quartile (red box), maximum (upper whisker) and minimum (lower whisker) of the population.

Live imaging of cortical microtubules. Cortical microtubules were analysed in epidermal cells of seedlings expressing mCherry-TUA5 (ref. 32) and AtEBla$\mathrm{GFP}^{35}$ or PIP5K3-YFP 37 . For movies of microtubule growth, $\mathrm{Z}$ stacks of planes intersecting the periclinal face of the cell $1 \mu \mathrm{m}$ apart $(0.51 \mu \mathrm{m}$ in elongating wildtype cells) were imaged every $5 \mathrm{~s}$ for $2.5 \mathrm{~min}$. Maximum intensity projections of each time point were used to assemble movies. For images of cortical microtubules in cells about to initiate outgrowth of a root hair, Z stacks of planes at $0.46 \mu \mathrm{m}$ distance intersecting the periclinal face of the cell were acquired and employed to generate maximum intensity projections. Relative PIP5K3-YFP position was determined measuring the distance between basal trichoblast membrane and basal end of PIP5K3-YFP patch on the upper cell face and dividing it by total cell length. Measurements were performed on $n=50$ cells per genotype from 39 to 41 roots.

Microtubule orientation measurements. Seedlings with wild-type or sab-5 phenotype expressing the atrichoblast marker pGL2:NTF ${ }^{54}$ and mCherry-TUA5 (ref. 32) were imaged live. Trichoblasts were identified by absence of pGL2:NTF. Z stacks of planes at $0.45 \mu \mathrm{m}$ distance were acquired on the periclinal face from either meristematic cells of $13-18 \mu \mathrm{m}$ or elongated cells of $26-36 \mu \mathrm{m}$ cell length. Maximum intensity projections were used to measure the angle of microtubules with respect to the root growth axis.

Generation of SAB-3xYpet and 3xYpet-SAB lines. A 3xYpet tag was fused to $\mathrm{SAB}$ at either the C- or the N-terminus by recombineering ${ }^{38}$. The JAtY clone 63C14, purchased from the John Innes Centre (Norwich, UK), was selected based on bacterial artificial chromosome end sequence information from the Arabidopsis thaliana Integrated Database, http://www.atidb.org/cgi-perl/gbrowse/atibrowse/. In this clone, the SAB-coding region is preceded and followed by 36 and $4.5 \mathrm{~kb}$ of gDNA, respectively. The JAtY transformation-competent bacterial artificial chromosome was transferred to Escherichia coli strain SW105, kindly provided by Dr N. Copeland (Mouse Cancer Genetics Program, National Cancer Institute Frederick, Frederick, MD, USA $)^{58}$. A recombineering cassette was generated by inserting the ampicillin resistance gene flanked by flippase recognition sites at the $3^{\prime}$-end of the codon-optimized $3 x Y$ pet $\operatorname{tag}^{38}$ (cassette available from the 
Arabidopsis Biological Resource Center, stock number CD3-1727). The cassette was amplified by PCR using two long recombineering primers (Supplementary Table S3): the forward primer included 50 nucleotoides homologous to the region upstream of the $3 \mathrm{x} Y$ pet insertion point (until right after the first codon of the $S A B$ gene for the N-terminal fusion and before the STOP codon for the C-terminal fusion); the reverse primer included 50 nucleotides homologous to the region downstream of the insertion point (ending just after the first codon or before the STOP codon of $S A B$, respectively). Cassette recombination into the JAtY clone was performed as described ${ }^{38}$, and the $A m p^{R}$ marker was removed on induction of the Flippase gene expression by arabinose. Constructs were sequenced and respective transformation-competent bacterial artificial chromosome clones transformed into Agrobacterium tumefaciens GV3101, which was used to transform sab-5 heterozygous Arabidopsis plants by floral dipping ${ }^{38,59}$. Transformants were selected by Basta and further screened by PCR, ensuring presence of the sab-5-mutant allele, of the tagged $S A B$ gene, and of the $S a c B$ gene (for primer sequences Supplementary Table S3). SacB lies close to the T-DNA left border and its presence confirmed transfer of at least one full copy of the T-DNA to the plant genome ${ }^{38} \cdot \mathrm{T}_{2}$ seedlings were analysed for fluorescence using a Zeiss LSM780 confocal microscope. Out of 12 SAB-3xYpet lines analysed, 7 displayed a very weak but identical fluorescent signal. Out of $103 \times$ Ypet-SAB lines, 9 showed the same pattern of fluorescence, indistinguishable from SAB-3xYpet lines. One line was selected for each construct and $\mathrm{T}_{2}$ plants homozygous for the sab-5 allele and the $S A B$ transgene were identified. Offspring of these plants was used for all further analyses and genetic crosses.

Plasmolysis. Plasmolysis was performed as described ${ }^{60}$. Seedlings were incubated in $1 \mathrm{ml}$ of $0.8 \mathrm{M}$ mannitol for $2 \mathrm{~h}$ under gentle agitation, stained for $30 \mathrm{~s}$ in $10 \mu \mathrm{g} \mathrm{ml}^{-1}$ propidium iodide in $0.8 \mathrm{M}$ mannitol, mounted on cover slips in the same medium and immediately imaged.

Generation of ER-ECFP-HDEL marker. The binary vector pGreenII0229 (ref. 61; obtained from the John Innes Centre, Norwich) was modified by adding a Cauliflower mosaic virus $35 \mathrm{~S}$ promoter (derived from the pSK36 vector ${ }^{62}$ ) and a Nopaline synthase terminator between the BamHI and SpeI, and at the NotI site of the multiple cloning site, respectively. The resulting vector was named p235S. ER signal peptide of Arabidopsis Basic chitinase (At3g12500) was amplified from gDNA using primers CHTTPFAscI and CHTTPRSpeI. PCR product was digested with $A s c \mathrm{I}$ and SpeI and cloned into the corresponding sites of the $\mathrm{p} 235 \mathrm{~S}$ vector, generating the p235S-TP construct. Enhanced cyan fluorescent protein (ECFP) coding sequence was amplified from the pECFP-Cl vector (Clontech) using primers CFPFSpeI and CFPERNotI, which introduced the coding sequence for the ER-retention signal peptide HDEL before the stop codon. PCR product was digested with SpeI and NotI and subcloned into the corresponding sites of the p235S-TP vector. After sequencing with 35SF and tNOSR primers, the resulting construct was transferred to A. tumefaciens strain $\mathrm{C} 58 \mathrm{C} 1$ and used to transform Arabidopsis Col-0 plants by floral dipping ${ }^{59}$. $\mathrm{T}_{1}$ transgenic plants were selected by Basta. Primer sequences are listed in Supplementary Table S3.

Visualization of SAB together with microtubules or F-actin. Seedlings expressing SAB-3xYpet or SAB-3xYpet and mCherry-TUA5 were fixed for $40 \mathrm{~min}$ in PME buffer (50 mM piperazine-1,4-bis(2-ethanesulfonic acid), $2 \mathrm{mM} \mathrm{MgSO}_{4}$, $5 \mathrm{mM}$ ethylene glycol-bis( $\beta$-aminoethylether)- $N, N, N^{\prime}, N^{\prime}$-tetraacetic acid, $\mathrm{pH}$ 6.9) supplemented with $0.1 \%$ Triton X-100 and $2 \%$ paraformaldehyde as described ${ }^{63}$. After three washes with PME buffer, seedlings were either mounted on cover slips in PME buffer and immediately imaged (for seedlings expressing SAB-3xYpet and mCherry-TUA5) or, for visualization of F-actin, mounted in $2 \%$ rhodamine phalloidin (Molecular Probes) in PME buffer and incubated $50 \mathrm{~min}$ in dark before imaging. To assess SAB-3xYpet localization relative to PPB, two sections (one through the middle of the cell and one $4-5 \mu \mathrm{m}$ apart) were evaluated.

Lambda scan of root hair initiation sites. Lambda scans of trichoblasts undergoing hair initiation were captured from live Col-0 seedlings and seedlings expressing SAB-3xYpet, using a Zeiss LSM780 confocal microscope. On excitation at $514 \mathrm{~nm}, 32$ images were acquired with narrow detection windows covering the spectral region between $416 \mathrm{~nm}$ and $686 \mathrm{~nm}$, in $\mathrm{Z}$ stacks of planes at a distance of $2 \mu \mathrm{m}$. Maximum intensity Z projections were generated and lambda stacks were visualized in wavelength-coded colour view employing ZEN2010 (Zeiss) software.

Statistical analysis. Positioning of root hairs, ROP or PIP5K3-YFP patches along trichoblasts, and cell division and microtubule orientation angles did not follow a normal distribution. Thus, significance of differences between distributions was tested with non-parametric, two-sample Kolmogorov-Smirnov test (http:// www.physics.csbsju.edu/stats/KS-test.n.plot_form.html), which considers both distributions' location and shape. Distributions of $n=150$ or 50 trichoblasts from root hair and ROP or PIP5K3-YFP position measurements, respectively, of $n=60$ cells per mitotic stage, and of $n=673-802$ or $578-520$ cortical microtubules were analysed with significance level $P<0.05$. Differences in trichome orientation were analysed by F-test (Microsoft Excel), with $n=174$. Measurements of GFP-BASL polar localization and trichome branching were analysed by Fisher's exact test (http://www.quantitativeskills.com/sisa/statistics/fiveby2.htm), with populations of $n>187$ and $n>400$, respectively, with significance level $P<0.05$. Analysis of trichoblast length was performed by two-tailed, unpaired $t$-test with equal variance (computed with Microsoft Excel), with $n=3$ average values of independent experiments and significance level $P<0.05$.

\section{References}

1. Goodrich, L. V. \& Strutt, D. Principles of planar polarity in animal development. Development 138, 1877-1892 (2011).

2. Bertet, C. \& Lecuit, T. Planar polarity and short-range polarization in Drosophila embryos. Semin. Cell Dev. Biol. 20, 1006-1013 (2009).

3. Masucci, J. D. \& Schiefelbein, J. W. The rhd6 mutation of Arabidopsis thaliana alters root-hair initiation through an auxin- and ethylene-associated process. Plant Physiol. 106, 1335-1346 (1994).

4. Fischer, U. et al. Vectorial information for Arabidopsis planar polarity is mediated by combined AUX1, EIN2, and GNOM activity. Curr. Biol. 16, 2143-2149 (2006).

5. Hülskamp, M., Misra, S. \& Jürgens, G. Genetic dissection of trichome cell development in Arabidopsis. Cell 76, 555-566 (1994).

6. Wu, J., Roman, A. C., Carvajal-Gonzalez, J. M. \& Mlodzik, M. Wg and Wnt4 provide long-range directional input to planar cell polarity orientation in Drosophila. Nat. Cell Biol. 15, 1045-1055 (2013).

7. Ikeda, Y. et al. Local auxin biosynthesis modulates gradient-directed planar polarity in Arabidopsis. Nat. Cell Biol. 11, 731-738 (2009).

8. Molendijk, A. J. et al. Arabidopsis thaliana Rop GTPases are localized to tips of root hairs and control polar growth. EMBO J. 20, 2779-2788 (2001).

9. Jones, M. A. et al. The Arabidopsis Rop2 GTPase is a positive regulator of both root hair initiation and tip growth. Plant Cell 14, 763-776 (2002).

10. Carol, R. J. et al. A RhoGDP dissociation inhibitor spatially regulates growth in root hair cells. Nature 438, 1013-1016 (2005).

11. Fu, Y., Xu, T., Zhu, L., Wen, M. \& Yang, Z. A ROP GTPase signaling pathway controls cortical microtubule ordering and cell expansion in Arabidopsis. Curr. Biol. 19, 1827-1832 (2009).

12. Xu, T. et al. Cell surface- and Rho GTPase-based auxin signaling controls cellular interdigitation in Arabidopsis. Cell 143, 99-110 (2010).

13. Ringli, C., Baumberger, N., Diet, A., Frey, B. \& Keller, B. ACTIN2 is essential for bulge site selection and tip growth during root hair development of arabidopsis. Plant Physiol. 129, 1464-1472 (2002).

14. Bao, Y., Kost, B. \& Chua, N. H. Reduced expression of alpha-tubulin genes in Arabidopsis thaliana specifically affects root growth and morphology, root hair development and root gravitropism. Plant J. 28, 145-157 (2001).

15. Kirik, V. et al. CLASP localizes in two discrete patterns on cortical microtubules and is required for cell morphogenesis and cell division in Arabidopsis. J. Cell Sci. 120, 4416-4425 (2007).

16. Ambrose, J. C., Shoji, T., Kotzer, A. M., Pighin, J. A. \& Wasteneys, G. O. The Arabidopsis CLASP gene encodes a microtubule-associated protein involved in cell expansion and division. Plant Cell 19, 2763-2775 (2007).

17. Dhonukshe, P. et al. A PLETHORA-auxin transcription module controls cell division plane rotation through MAP65 and CLASP. Cell 149, 383-396 (2012).

18. Ambrose, C. et al. CLASP interacts with Sorting Nexin 1 to link microtubules and auxin transport via PIN2 recycling in Arabidopsis thaliana. Dev. Cell 24, 649-659 (2013).

19. Kakar, K., Zhang, H., Scheres, B. \& Dhonukshe, P. CLASP-mediated cortical microtubule organization guides PIN polarization axis. Nature 495, 529-533 (2013).

20. Galjart, N. CLIPs. and CLASPs and cellular dynamics. Nat. Rev. Mol. Cell Biol. 6, 487-498 (2005).

21. Ambrose, C., Allard, J. F., Cytrynbaum, E. N. \& Wasteneys, G. O. A CLASPmodulated cell edge barrier mechanism drives cell-wide cortical microtubule organization in Arabidopsis. Nat. Commun. 2, 430 (2011).

22. Strader, L. C. \& Bartel, B. The Arabidopsis PLEIOTROPIC DRUG RESISTANCE8/ABCG36 ATP binding cassette transporter modulates sensitivity to the auxin precursor indole-3-butyric acid. Plant Cell 21, 1992-2007 (2009).

23. Langowski, L., Ruzicka, K., Naramoto, S., Kleine-Vehn, J. \& Friml, J. Trafficking to the outer polar domain defines the root-soil interface. Curr. Biol. 20, 904-908 (2010).

24. Dong, J., MacAlister, C. A. \& Bergmann, D. C. BASL controls asymmetric cell division in Arabidopsis. Cell 137, 1320-1330 (2009).

25. Buschmann, H. \& Lloyd, C. W. Arabidopsis mutants and the network of microtubule-associated functions. Mol. Plant 1, 888-898 (2008).

26. Benfey, P. N. et al. Root development in Arabidopsis: four mutants with dramatically altered root morphogenesis. Development 119, 57-70 (1993). 
27. Aeschbacher, R. A., Hauser, M. T., Feldmann, K. A. \& Benfey, P. N. The SABRE gene is required for normal cell expansion in Arabidopsis. Genes Dev. 9, 330-340 (1995).

28. Procissi, A. et al. KINKY POLLEN encodes a SABRE-like protein required for tip growth in Arabidopsis and conserved among eukaryotes. Plant J. 36, 894-904 (2003).

29. Yu, H., Luo, N., Sun, L. \& Liu, D. HPS4/SABRE regulates plant responses to phosphate starvation through antagonistic interaction with ethylene signalling. J. Exp. Bot. 63, 4527-4538 (2012).

30. Xu, Z. \& Dooner, H. K. The Maize aberrant pollen transmission 1 gene is a $S A B R E / K I P$ homolog required for pollen tube growth. Genetics 172, 1251-1261 (2006).

31. Marchler-Bauer, A. et al. CDD: conserved domains and protein threedimensional structure. Nucleic Acids Res. 41, D348-D352 (2013).

32. Gutierrez, R., Lindeboom, J. J., Paredez, A. R., Emons, A. M. \& Ehrhardt, D. W. Arabidopsis cortical microtubules position cellulose synthase delivery to the plasma membrane and interact with cellulose synthase trafficking compartments. Nat. Cell Biol. 11, 797-806 (2009)

33. Sampathkumar, A. et al. Live cell imaging reveals structural associations between the actin and microtubule cytoskeleton in Arabidopsis. Plant Cell 23, 2302-2313 (2011).

34. Boisnard-Lorig, C. et al. Dynamic analyses of the expression of the HISTONE::YFP fusion protein in Arabidopsis show that syncytial endosperm is divided in mitotic domains. Plant Cell 13, 495-509 (2001)

35. Chan, J., Calder, G. M., Doonan, J. H. \& Lloyd, C. W. EB1 reveals mobile microtubule nucleation sites in Arabidopsis. Nat. Cell Biol. 5, 967-971 (2003).

36. Sambade, A., Pratap, A., Buschmann, H., Morris, R. J. \& Lloyd, C. The influence of light on microtubule dynamics and alignment in the Arabidopsis hypocotyl. Plant Cell 24, 192-201 (2012).

37. Kusano, H. et al. The Arabidopsis phosphatidylinositol phosphate 5-kinase PIP5K3 is a key regulator of root hair tip growth. Plant Cell 20, 367-380 (2008).

38. Zhou, R., Benavente, L. M., Stepanova, A. N. \& Alonso, J. M. A recombineering-based gene tagging system for Arabidopsis. Plant J. 66, 712-723 (2011)

39. Zheng, H., Kunst, L., Hawes, C. \& Moore, I. A GFP-based assay reveals a role for RHD3 in transport between the endoplasmic reticulum and Golgi apparatus. Plant J. 37, 398-414 (2004).

40. Swarup, R. et al. Structure-function analysis of the presumptive arabidopsis auxin permease AUX1. Plant Cell 16, 3069-3083 (2004).

41. Geldner, N. et al. Rapid, combinatorial analysis of membrane compartments in intact plants with a multicolor marker set. Plant J. 59, 169-178 (2009).

42. von der Fecht-Bartenbach, J. et al. Function of the anion transporter AtCLC-d in the trans-Golgi network. Plant J. 50, 466-474 (2007).

43. Benschop, J. J. et al. Quantitative phosphoproteomics of early elicitor signaling in Arabidopsis. Mol. Cell Proteomics 6, 1198-1214 (2007).

44. Doonan, J. H., Cove, D. J. \& Lloyd, C. W. Immunofluorescence microscopy of microtubules in intact cell lineages of the moss, Physcomitrella patens. I. Normal and CIPC-treated tip cells. J. Cell Sci. 75, 131-147 (1985).

45. Anderhag, P., Hepler, P. K. \& Lazzaro, M. D. Microtubules and microfilaments are both responsible for pollen tube elongation in the conifer Picea abies (Norway spruce). Protoplasma 214, 141-157 (2000).

46. Rounds, C. M. \& Bezanilla, M. Growth mechanisms in tip-growing plant cells. Annu. Rev. Plant Biol. 64, 243-265 (2013).

47. Bibikova, T. N., Blancaflor, E. B. \& Gilroy, S. Microtubules regulate tip growth and orientation in root hairs of Arabidopsis thaliana. Plant J. 17, 657-665 (1999).

48. Song, J., Yang, W., Shih Ie, M., Zhang, Z. \& Bai, J. Identification of BCOX1, a novel gene overexpressed in breast cancer. Biochim. Biophys. Acta 1760, 62-69 (2006).

49. Zhou, F. L., Zhang, W. G., Meng, X., Chen, G. \& Wang, J. L. Bioinformatic analysis and identification for a novel antigen MLAA-22 in acute monocytic leukemia. Zhongguo. Shi Yan Xue Ye Xue Za Zhi 16, 466-471 (2008).

50. Skog, J. et al. Glioblastoma microvesicles transport RNA and proteins that promote tumour growth and provide diagnostic biomarkers. Nat. Cell Biol. 10, 1470-1476 (2008)

51. Hong, B. S. et al. Colorectal cancer cell-derived microvesicles are enriched in cell cycle-related mRNAs that promote proliferation of endothelial cells. BMC Genomics 10, 556 (2009).

52. Alonso, J. M. et al. Genome-wide insertional mutagenesis of Arabidopsis thaliana. Science 301, 653-657 (2003).
53. Stein, M. et al. Arabidopsis PEN3/PDR8, an ATP binding cassette transporter, contributes to nonhost resistance to inappropriate pathogens that enter by direct penetration. Plant Cell 18, 731-746 (2006).

54. Deal, R. B. \& Henikoff, S. A simple method for gene expression and chromatin profiling of individual cell types within a tissue. Dev. Cell 18, 1030-1040 (2010).

55. Müller, A. et al. AtPIN2 defines a locus of Arabidopsis for root gravitropism control. EMBO J. 17, 6903-6911 (1998).

56. Lauber, M. H. et al. The Arabidopsis KNOLLE protein is a cytokinesis-specific syntaxin. J. Cell Biol. 139, 1485-1493 (1997).

57. Lukowitz, W., Gillmor, C. S. \& Scheible, W.-R. Positional cloning in Arabidopsis. Why it feels good to have a genome initiative working for you. Plant Physiol. 123, 795-805 (2000).

58. Warming, S., Costantino, N., Court, D. L., Jenkins, N. A. \& Copeland, N. G. Simple and highly efficient BAC recombineering using galK selection. Nucleic Acids Res. 33, e36 (2005).

59. Clough, S. J. \& Bent, A. F. Floral dip: a simplified method for Agrobacteriummediated transformation of Arabidopsis thaliana. Plant J. 16, 735-743 (1998).

60. Gagne, J. M. \& Clark, S. E. The Arabidopsis stem cell factor POLTERGEIST is membrane localized and phospholipid stimulated. Plant Cell 22, 729-743 (2010).

61. Hellens, R. P., Edwards, E. A., Leyland, N. R., Bean, S. \& Mullineaux, P. M. pGreen: a versatile and flexible binary Ti vector for Agrobacterium-mediated plant transformation. Plant Mol. Bio. 42, 819-832 (2000).

62. Ikeda, Y., Banno, H., Niu, Q. W., Howell, S. H. \& Chua, N. H. The ENHANCER OF SHOOT REGENERAION 2 gene in Arabidopsis regulates CUP-SHAPED COTYLEDON 1 at the transcriptional level and controls cotyledon development. Plant Cell Physiol. 47, 1443-1456 (2006)

63. Nishimura, T., Yokota, E., Wada, T., Shimmen, T. \& Okada, K. An Arabidopsis ACT2 dominant-negative mutation, which disturbs F-actin polymerization, reveals its distinctive function in root development. Plant Cell Physiol. 44, 1131-1140 (2003).

\section{Acknowledgements}

We are grateful to C. Ambrose, T. Aoyama, F. Berger, D.C. Bergmann, J.R. Ecker, D.W. Ehrhardt, N. Geldner, T. Hashimoto, S. Hennikoff, K. Palme, S. Persson, C.W. Lloyd, A. Sampathkumar, B. Scheres, K. Schumacher, S. Somerville and G.O. Wasteneys for sharing published materials and reagents. We thank the Nottingham Arabidopsis Stock Centre for providing seed stocks. This work was supported by Swedish Research Council (Vetenskapsrådet) Grant numbers 2006-522 and 2009-4846, and the Knut \& Alice Wallenberg foundation project grant 'ShapeSystems' to M.G., NSF Grant DBI0820755 to J.M.A. and NSF Grant MCB0923727 to J.M.A. and A.N.S

\section{Author contributions}

M.G. conceived the project; M.G. and S.P. designed most of the experiments, S.P. performed the majority of the experiments; S.P., A.G., C.K., Y.I., L.K., P.H. and M.G. designed, performed and analysed experiments apart from recombineering work that was designed, performed and analysed by S.P., A.N.S. and J.M.A. M.G., S.P., A.G., C.K. and L.K. analysed and interpreted the results, A.N.S. and J.M.A. provided unpublished materials, valuable experimental advise, supervised and hosted S.P. during recombineering work. All authors contributed to the interpretation of results. M.G. and S.P. wrote the manuscript. All authors commented on and edited the manuscript.

\section{Additional information}

Supplementary Information accompanies this paper at http://www.nature.com/ naturecommunications

Competing financial interests: The authors declare no competing financial interests.

Reprints and permission information is available online at http://npg.nature.com/ reprintsandpermissions/

How to cite this article: Pietra, S. et al. Arabidopsis SABRE and CLASP interact to stabilize cell division plane orientation and planar polarity. Nat. Commun. 4:2779 doi: $10.1038 /$ ncomms3779 (2013).

This work is licensed under a Creative Commons AttributionNonCommercial-ShareAlike 3.0 Unported License. To view a copy of this license, visit http://creativecommons.org/licenses/by-nc-sa/3.0/ 